\title{
The existence, uniqueness and asymptotic estimates of solutions for third-order full nonlinear singularly perturbed vector boundary value problems
}

\author{
Xiaojie Lin ${ }^{1 *}$, Jiang Liu and Can Wang
}

${ }^{*}$ Correspondence:

linxiaojie1973@163.com

1 School of Mathematics and

Statistics, Jiangsu Normal University,

Xuzhou, P.R. China

\section{Springer}

\begin{abstract}
In this paper, we discuss third-order full nonlinear singularly perturbed vector boundary value problems. We first present the existence of solutions for the nonlinear vector boundary value problems without perturbation by using the upper and lower solutions method and topological degree theory. Then the existence, uniqueness and asymptotic estimates of solutions for the singularly perturbed vector boundary value problems are established by constructing appropriate a lower solution-upper solution pair, as well as analysis technique. Some known results are extended.
\end{abstract}

MSC: 34D15; 34E10; 34B15

Keywords: Vector boundary value problems; Singular perturbation; Existence and uniqueness; Upper and lower solutions; Asymptotic estimates

\section{Introduction}

In the past few decades, nonlinear boundary value problems (BVPs) and singularly perturbed boundary value problems (SPBVPs) have been studied widely [1-11]. For example, Zhao [5] discussed the existence and asymptotic estimates of the solutions for a thirdorder boundary value problem with perturbation. Du et al. [9] were concerned with a more generalized third-order singularly perturbed differential equations with multi-point boundary conditions and obtained the existence and uniqueness as well as the asymptotic estimates of solutions. Lodhi and Mishra [12] discussed second order singularly perturbed nonlinear boundary value problems by using the quintic B-spline method. Recently, the geometric singular perturbation theory has also received a great deal of interests in studying the Burgers-KdV equation [13], the vector-disease model [14], the perturbed BBM equation [15], the perturbed Camassa-Holm equation [16] and the perturbed shallow water wave model [17] etc.

However, the boundary value problems in the above-mentioned references are all scalar and little work has been published for vector systems [18-20]. Motivated by the above work, in this article, we discuss the singular perturbations of third-order nonlinear differ-

(c) The Author(s) 2020. This article is licensed under a Creative Commons Attribution 4.0 International License, which permits use, sharing, adaptation, distribution and reproduction in any medium or format, as long as you give appropriate credit to the original author(s) and the source, provide a link to the Creative Commons licence, and indicate if changes were made. The images or other third party material in this article are included in the article's Creative Commons licence, unless indicated otherwise in a credit line to the material. If material is not included in the article's Creative Commons licence and your intended use is not permitted by statutory regulation or exceeds the permitted use, you will need to obtain permission directly from the copyright holder. To view a copy of this licence, visit http://creativecommons.org/licenses/by/4.0/. 
ential system

$$
\varepsilon \mathbf{x}^{\prime \prime \prime}(t)+\mathbf{F}\left(t, \mathbf{x}(t), \mathbf{x}^{\prime}(t), \mathbf{x}^{\prime \prime}(t), \varepsilon\right)=\mathbf{0}, \quad 0 \leq t \leq 1,0<\varepsilon \ll 1,
$$

with full nonlinear multi-point boundary value conditions

$$
\left\{\begin{array}{l}
\mathbf{x}(0, \varepsilon)=\mathbf{0} \\
\mathbf{G}\left(\mathbf{x}^{\prime}(0, \varepsilon), \mathbf{x}^{\prime \prime}(0, \varepsilon), \mathbf{x}\left(\xi_{1}, \varepsilon\right), \mathbf{x}\left(\xi_{2}, \varepsilon\right), \ldots, \mathbf{x}\left(\xi_{m-2}, \varepsilon\right)\right)=\mathbf{A} \\
\mathbf{H}\left(\mathbf{x}^{\prime}(1, \varepsilon), \mathbf{x}^{\prime \prime}(1, \varepsilon), \mathbf{x}\left(\eta_{1}, \varepsilon\right), \mathbf{x}\left(\eta_{2}, \varepsilon\right), \ldots, \mathbf{x}\left(\eta_{n-2}, \varepsilon\right)\right)=\mathbf{B}
\end{array}\right.
$$

where $\mathbf{x}=\left(x_{1}, x_{2}, \ldots, x_{N}\right)^{T}, \mathbf{F}\left(t, \mathbf{x}, \mathbf{x}^{\prime}, \mathbf{x}^{\prime \prime}, \varepsilon\right)=\left(f_{1}, f_{2}, \ldots, f_{N}\right)^{T} \in R^{N}, f_{i}=f_{i}\left(t, \mathbf{x}, \mathbf{x}^{\prime}, \mathbf{x}^{\prime \prime}, \varepsilon\right) \in R$, $\mathbf{G}\left(\mathbf{x}^{\prime}(0, \varepsilon), \mathbf{x}^{\prime \prime}(0, \varepsilon), \mathbf{x}\left(\xi_{1}, \varepsilon\right), \ldots, \mathbf{x}\left(\xi_{m-2}, \varepsilon\right)\right)=\left(g_{1}, g_{2}, \ldots, g_{N}\right)^{T} \in R^{N}, g_{i}=g_{i}\left(\mathbf{x}^{\prime}(0, \varepsilon), \mathbf{x}^{\prime \prime}(0, \varepsilon)\right.$, $\left.\mathbf{x}\left(\xi_{1}, \varepsilon\right), \ldots, \mathbf{x}\left(\xi_{m-2}, \varepsilon\right)\right) \in R, \mathbf{H}\left(\mathbf{x}^{\prime}(1, \varepsilon), \mathbf{x}^{\prime \prime}(1, \varepsilon), \mathbf{x}\left(\eta_{1}, \varepsilon\right), \ldots, \mathbf{x}\left(\eta_{n-2}, \varepsilon\right)\right)=\left(h_{1}, h_{2}, \ldots, h_{N}\right)^{T} \in$ $R^{N}, h_{i}=h_{i}\left(\mathbf{x}^{\prime}(1, \varepsilon), \mathbf{x}^{\prime \prime}(1, \varepsilon), \mathbf{x}\left(\eta_{1}, \varepsilon\right), \ldots, \mathbf{x}\left(\eta_{n-2}, \varepsilon\right)\right) \in R, i=1,2, \ldots, N, \mathbf{A}=\left(A_{1}, A_{2}, \ldots, A_{N}\right)^{T}$, $\mathbf{B}=\left(B_{1}, B_{2}, \ldots, B_{N}\right)^{T} \in R^{N}, 0<\xi_{1}<\xi_{2}<\cdots<\xi_{m-2}<1,0<\eta_{1}<\eta_{2}<\cdots<\eta_{n-2}<1, \varepsilon$ is a small positive parameter.

In order to study SPBVP (1.1), (1.2), we need to study the following nonlinear unperturbed vector multi-point boundary value problem:

$$
\begin{aligned}
& \mathbf{x}^{\prime \prime \prime}(t)+\mathbf{F}\left(t, \mathbf{x}(t), \mathbf{x}^{\prime}(t), \mathbf{x}^{\prime \prime}(t)\right)=\mathbf{0}, \quad 0 \leq t \leq 1, \\
& \left\{\begin{array}{l}
\mathbf{x}(0)=\mathbf{0}, \\
\mathbf{G}\left(\mathbf{x}^{\prime}(0), \mathbf{x}^{\prime \prime}(0), \mathbf{x}\left(\xi_{1}\right), \mathbf{x}\left(\xi_{2}\right), \ldots, \mathbf{x}\left(\xi_{m-2}\right)\right)=\mathbf{A}, \\
\mathbf{H}\left(\mathbf{x}^{\prime}(1), \mathbf{x}^{\prime \prime}(1), \mathbf{x}\left(\eta_{1}\right), \mathbf{x}\left(\eta_{2}\right), \ldots, \mathbf{x}\left(\eta_{n-2}\right)\right)=\mathbf{B} .
\end{array}\right.
\end{aligned}
$$

The remaining part of this paper is organized as follows. In Sect. 2, we present some definitions and lemmas. In Sect. 3, we obtain the existence of solutions for BVP (1.3), (1.4) by using the differential inequality technique and topological degree theory. Furthermore, we give the existence and asymptotic estimates of solutions of SPBVP (1.1), (1.2). In Sect. 4, we establish the uniqueness result of SPBVP (1.1), (1.2).

\section{Preliminaries}

For the simplicity, for $\forall \mathbf{x}=\left(x_{1}, \ldots, x_{N}\right)^{T}, \mathbf{y}=\left(y_{1}, \ldots, y_{N}\right)^{T} \in R^{N}$, we denote $\mathbf{x} \preceq \mathbf{y}(\mathbf{x} \prec \mathbf{y})$, if and only if $x_{i} \leq y_{i}\left(x_{i}<y_{i}\right), i=1,2, \ldots, N$. Similarly, we can define $\mathbf{x} \succeq \mathbf{y}(\mathbf{x} \succ \mathbf{y})$. We use the norm $\|\mathbf{x}\|=\left(\sum_{i=1}^{N} x_{i}^{2}\right)^{\frac{1}{2}}$, for $\forall \mathbf{x}=\left(x_{1}, \ldots, x_{N}\right) \in R^{N}$.

Definition 1 The vector function $\mathbf{F}\left(t, \mathbf{x}_{1}, \mathbf{x}_{2}, \mathbf{x}_{3}\right) \in R^{N}$ is increasing in $\mathbf{x}_{1}$, if for $\forall \mathbf{y}_{1} \succeq \mathbf{x}_{1}$, such that

$$
\mathbf{F}\left(t, \mathbf{y}_{1}, \mathbf{x}_{2}, \mathbf{x}_{3}\right) \succeq \mathbf{F}\left(t, \mathbf{x}_{1}, \mathbf{x}_{2}, \mathbf{x}_{3}\right) .
$$

The vector function $\mathbf{G}\left(\mathbf{x}_{1}, \mathbf{x}_{2}, \ldots, \mathbf{x}_{m}\right) \in R^{N}$ is increasing in $\mathbf{x}_{k}, k=1,2, \ldots, m$, if, for $\forall \mathbf{y}_{k} \succeq$ $\mathbf{x}_{k}$,

$$
\mathbf{G}\left(\mathbf{x}_{1}, \mathbf{x}_{2}, \ldots, \mathbf{x}_{k-1}, \mathbf{y}_{k}, \mathbf{x}_{k+1}, \ldots, \mathbf{x}_{m}\right) \succeq \mathbf{G}\left(\mathbf{x}_{1}, \mathbf{x}_{2}, \ldots, \mathbf{x}_{k-1}, \mathbf{x}_{k}, \mathbf{x}_{k+1}, \ldots, \mathbf{x}_{m}\right)
$$

The vector function $\mathbf{H}\left(\mathbf{x}_{1}, \mathbf{x}_{2}, \ldots, \mathbf{x}_{n}\right) \in R^{N}$ is decreasing in $\mathbf{x}_{j}, j=1,2, \ldots, n$, if, for $\forall \mathbf{y}_{j} \succeq \mathbf{x}_{j}$,

$$
\mathbf{H}\left(\mathbf{x}_{1}, \mathbf{x}_{2}, \ldots, \mathbf{x}_{j-1}, \mathbf{y}_{j}, \mathbf{x}_{j+1}, \ldots, \mathbf{x}_{n}\right) \preceq \mathbf{H}\left(\mathbf{x}_{1}, \mathbf{x}_{2}, \ldots, \mathbf{x}_{j-1}, \mathbf{x}_{j}, \mathbf{x}_{j+1}, \ldots, \mathbf{x}_{n}\right) .
$$


Similarly, we define the case that $\mathbf{G}\left(\mathbf{x}_{1}, \mathbf{x}_{2}, \ldots, \mathbf{x}_{m}\right)$ is decreasing in $\mathbf{x}_{k}, k=1,2, \ldots, m$. $\mathbf{H}\left(\mathbf{x}_{1}, \mathbf{x}_{2}, \ldots, \mathbf{x}_{n}\right)$ is increasing in $\mathbf{x}_{j}, j=1,2, \ldots, n$.

Definition 2 We define a function $\delta$ as follows:

$$
\delta\left(\mathbf{z}_{1}, \mathbf{z}_{2}, \mathbf{z}_{3}\right)= \begin{cases}\mathbf{z}_{1}, & \mathbf{z}_{2} \prec \mathbf{z}_{1}, \\ \mathbf{z}_{2}, & \mathbf{z}_{1} \preceq \mathbf{z}_{2} \preceq \mathbf{z}_{3}, \\ \mathbf{z}_{3}, & \mathbf{z}_{2} \succ \mathbf{z}_{3},\end{cases}
$$

where $\mathbf{z}_{v}=\left(z_{v 1}, z_{v 2}, \ldots, z_{v N}\right)^{T} \in R^{N}, v=1,2,3, \mathbf{z}_{1} \preceq \mathbf{z}_{3}$.

Definition 3 ([20]) $\mathbf{F}(t, \mathbf{x}, \mathbf{y}, \mathbf{z})$ is said to satisfy Nagumo condition with respect to $\mathbf{z}$, for $(t, \mathbf{x}, \mathbf{y}, \mathbf{z}) \in[0,1] \times R^{3 N}$, if $\mathbf{F}(t, \mathbf{x}, \mathbf{y}, \mathbf{z})$ satisfies one of the following conditions:

(i) There exist nondecreasing functions $\Phi_{i} \in C([0,+\infty),(0,+\infty)), i=1,2, \ldots, N$, such that

$$
\left|f_{i}(t, \mathbf{x}, \mathbf{y}, \mathbf{z})\right| \leq \Phi_{i}\left(\left|z_{i}\right|\right) \text { and } \quad \int_{0}^{+\infty} \frac{s d s}{\Phi_{i}(s)}=+\infty
$$

(ii) There exist nondecreasing functions $\Phi \in C([0,+\infty),(0,+\infty))$, such that

$$
\|\mathbf{F}(t, \mathbf{x}, \mathbf{y}, \mathbf{z})\| \leq \Phi(\|\mathbf{z}\|) \quad \text { and } \quad \frac{s^{2}}{\Phi(s)}=+\infty, \quad s \rightarrow+\infty
$$

Definition $4([10,20])$ A vector function $\alpha(t)=\left(\alpha_{1}(t), \ldots, \alpha_{N}(t)\right)^{T} \in C^{3}\left([0,1], R^{N}\right)$ is called a lower solution of BVP (1.3), (1.4), if for $i=1,2, \ldots, N$,

$$
\alpha_{i}^{\prime \prime \prime}(t)+f_{i}\left(t, \mathbf{x}_{\alpha_{i}}(t), \mathbf{x}_{\alpha_{i}}^{\prime}(t), \mathbf{x}_{\alpha_{i}}^{\prime \prime}(t)\right) \geq 0, \quad 0 \leq t \leq 1
$$

and

$$
\begin{aligned}
& \alpha_{i}(0) \leq 0, \\
& g_{i}\left(\boldsymbol{\alpha}^{\prime}(0), \boldsymbol{\alpha}^{\prime \prime}(0), \boldsymbol{\alpha}\left(\xi_{1}\right), \ldots, \boldsymbol{\alpha}\left(\xi_{m-2}\right)\right) \leq A_{i}, \\
& h_{i}\left(\boldsymbol{\alpha}^{\prime}(1), \boldsymbol{\alpha}^{\prime \prime}(1), \boldsymbol{\alpha}\left(\eta_{1}\right), \ldots, \boldsymbol{\alpha}\left(\eta_{n-2}\right)\right) \leq B_{i} .
\end{aligned}
$$

Similarly, a vector function $\boldsymbol{\beta}(t)=\left(\beta_{1}(t), \ldots, \beta_{N}(t)\right)^{T} \in C^{3}\left([0,1], R^{N}\right)$ is called an upper solution of BVP (1.3), (1.4), if for $i=1,2, \ldots, N$,

$$
\beta_{i}^{\prime \prime \prime}(t)+f_{i}\left(t, \mathbf{x}_{\beta_{i}}(t), \mathbf{x}_{\beta_{i}}^{\prime}(t), \mathbf{x}_{\beta_{i}}^{\prime \prime}(t)\right) \leq 0, \quad 0 \leq t \leq 1
$$

and

$$
\begin{aligned}
& \beta_{i}(0) \geq 0, \\
& g_{i}\left(\boldsymbol{\beta}^{\prime}(0), \boldsymbol{\beta}^{\prime \prime}(0), \boldsymbol{\beta}\left(\xi_{1}\right), \ldots, \boldsymbol{\beta}\left(\xi_{m-2}\right)\right) \geq A_{i}, \\
& h_{i}\left(\boldsymbol{\beta}^{\prime}(1), \boldsymbol{\beta}^{\prime \prime}(1), \boldsymbol{\beta}\left(\eta_{1}\right), \ldots, \boldsymbol{\beta}\left(\eta_{n-2}\right)\right) \geq B_{i},
\end{aligned}
$$


where

$$
\begin{aligned}
& \mathbf{x}_{\alpha_{i}}=\left(x_{1}, \ldots, x_{i-1}, \alpha_{i}, x_{i+1}, \ldots, x_{N}\right), \\
& \mathbf{x}_{\alpha_{i}}^{\prime}=\left(x_{1}^{\prime}, \ldots, x_{i-1}^{\prime}, \alpha_{i}^{\prime}, x_{i+1}^{\prime}, \ldots, x_{N}^{\prime}\right), \\
& \mathbf{x}_{\alpha_{i}}^{\prime \prime}=\left(x_{1}^{\prime \prime}, \ldots, x_{i-1}^{\prime \prime}, \alpha_{i}^{\prime \prime}, x_{i+1}^{\prime \prime}, \ldots, x_{N}^{\prime \prime}\right),
\end{aligned}
$$

$\mathbf{x}_{\beta_{i}}, \mathbf{x}_{\beta_{i}}^{\prime}, \mathbf{x}_{\beta_{i}}^{\prime \prime}$ are defined analogously.

Similar to $[10,20]$, we have Lemma 2.1 and we omit the proof.

Lemma 2.1 Assume that $\rho_{s}(t, \varepsilon)=\operatorname{diag}\left(\rho_{s 1}(t, \varepsilon), \ldots, \rho_{s N}(t, \varepsilon)\right) \in C\left([0,1] \times\left[0, \varepsilon_{0}\right], R^{N \times N}\right), s=$ $1,2,3, \rho_{3 i}(t, \varepsilon) \geq 0,(t, \varepsilon) \in[0,1] \times\left[0, \varepsilon_{0}\right]$ and there exists $\boldsymbol{\beta}(t, \varepsilon)=\left(\beta_{1}(t, \varepsilon), \ldots, \beta_{N}(t, \varepsilon)\right)^{T} \in$ $C^{3}\left([0,1] \times\left[0, \varepsilon_{0}\right], R^{N}\right)$, such that $\boldsymbol{\beta}^{\prime}(t, \varepsilon) \succ \mathbf{0}$ and

$$
\begin{aligned}
& \varepsilon \boldsymbol{\beta}^{\prime \prime \prime}(t, \varepsilon)+\rho_{1}(t, \varepsilon) \boldsymbol{\beta}^{\prime \prime}(t, \varepsilon)+\rho_{2}(t, \varepsilon) \boldsymbol{\beta}^{\prime}(t, \varepsilon)+\rho_{3}(t, \varepsilon) \boldsymbol{\beta}(t, \varepsilon) \prec \mathbf{0}, \quad 0 \leq t \leq 1, \\
& \left\{\begin{array}{l}
\boldsymbol{\beta}(0, \varepsilon) \succeq \mathbf{0}, \\
P_{1} \boldsymbol{\beta}^{\prime}(0, \varepsilon)+Q_{1} \boldsymbol{\beta}^{\prime \prime}(0, \varepsilon)+\sum_{k=1}^{m-2} \mu_{k} \boldsymbol{\beta}\left(\xi_{k}, \varepsilon\right) \succ \mathbf{0}, \\
P_{2} \boldsymbol{\beta}^{\prime}(1, \varepsilon)+Q_{2} \boldsymbol{\beta}^{\prime \prime}(1, \varepsilon)+\sum_{j=1}^{n-2} v_{j} \boldsymbol{\beta}\left(\eta_{j}, \varepsilon\right) \succ \mathbf{0},
\end{array}\right.
\end{aligned}
$$

where $P_{l}=\operatorname{diag}\left(p_{l 1}, p_{l 2}, \ldots, p_{l N}\right), Q_{l}=\operatorname{diag}\left(q_{l 1}, q_{l 2}, \ldots, q_{l N}\right), l=1,2, \mu_{k}=\operatorname{diag}\left(\mu_{k 1}, \ldots, \mu_{k N}\right)$, $v_{j}=\operatorname{diag}\left(v_{j 1}, \ldots, v_{j N}\right)$ satisfy $q_{1 i} \leq 0, q_{2 i} \geq 0, \mu_{k i} \leq 0, v_{j i} \leq 0, i=1,2, \ldots, N, k=1,2, \ldots, m-2$, $j=1,2, \ldots, n-2$.

Then the singularly perturbed boundary value problem

$$
\begin{aligned}
& \varepsilon \mathbf{x}^{\prime \prime \prime}(t, \varepsilon)+\rho_{1}(t, \varepsilon) \mathbf{x}^{\prime \prime}(t, \varepsilon)+\rho_{2}(t, \varepsilon) \mathbf{x}^{\prime}(t, \varepsilon)+\rho_{3}(t, \varepsilon) \mathbf{x}(t, \varepsilon)=\mathbf{0}, \quad 0 \leq t \leq 1, \\
& \left\{\begin{array}{l}
\mathbf{x}(0, \varepsilon)=\mathbf{0}, \\
P_{1} \mathbf{x}^{\prime}(0, \varepsilon)+Q_{1} \mathbf{x}^{\prime \prime}(0, \varepsilon)+\sum_{k=1}^{m-2} \mu_{k} \mathbf{x}\left(\xi_{k}, \varepsilon\right)=\mathbf{0}, \\
P_{2} \mathbf{x}^{\prime}(1, \varepsilon)+Q_{2} \mathbf{x}^{\prime \prime}(1, \varepsilon)+\sum_{j=1}^{n-2} v_{j} \mathbf{x}\left(\eta_{j}, \varepsilon\right)=\mathbf{0},
\end{array}\right.
\end{aligned}
$$

has only a zero solution.

\section{Existence results}

\subsection{Existence result of the modified problem}

Assume that $\boldsymbol{\alpha}(t)=\left(\alpha_{1}(t), \ldots, \alpha_{N}(t)\right)^{T}, \boldsymbol{\beta}(t)=\left(\beta_{1}(t), \ldots, \beta_{N}(t)\right)^{T} \in C^{3}\left([0,1], R^{N}\right), \boldsymbol{\alpha}(t) \preceq$ $\boldsymbol{\beta}(t), \boldsymbol{\alpha}^{\prime}(t) \preceq \boldsymbol{\beta}^{\prime}(t), 0 \leq t \leq 1$. We define the modified function as

$$
\overline{\mathbf{F}}\left(t, \mathbf{x}, \mathbf{x}^{\prime}, \mathbf{x}^{\prime \prime}\right)=\mathbf{F}\left(t, \overline{\mathbf{x}}, \overline{\mathbf{x}}^{\prime}, \overline{\mathbf{x}}^{\prime \prime}\right)-\omega\left(\mathbf{x}^{\prime}\right)
$$

where

$$
\begin{aligned}
& \overline{\mathbf{x}}(t)=\delta\left(\mathbf{x}_{\alpha_{i}}(t), \mathbf{x}(t), \mathbf{x}_{\beta_{i}}(t)\right), \\
& \overline{\mathbf{x}}^{\prime}(t)=\delta\left(\mathbf{x}_{\alpha_{i}}^{\prime}(t), \mathbf{x}^{\prime}(t), \mathbf{x}_{\beta_{i}}^{\prime}(t)\right), \\
& \overline{\mathbf{x}}^{\prime \prime}(t)=\delta\left(-\mathbf{D}, \mathbf{x}^{\prime \prime}(t), \mathbf{D}\right),
\end{aligned}
$$


$\mathbf{D}=\left(D_{1}, \ldots, D_{N}\right)^{T} \in R^{N}$ is a positive constant vector, such that

$$
\begin{aligned}
& D_{i}>\max _{t \in I}\left\{2 M_{i},\left|\alpha_{i}^{\prime \prime}(t)\right|,\left|\beta_{i}^{\prime \prime}(t)\right|\right\} \text { and } \int_{2 M_{i}}^{D_{i}} \frac{s d s}{\Phi_{i}(s)}>2 M_{i}, \\
& M_{i}>\max _{t \in I}\left\{\left|\alpha_{i}^{\prime}(t)\right|,\left|\beta_{i}^{\prime}(t)\right|\right\}, \quad i=1,2, \ldots, N .
\end{aligned}
$$

$\omega\left(\mathbf{x}^{\prime}\right)$ is continuous and bounded, satisfying

$$
\omega\left(\mathbf{x}^{\prime}\right) \begin{cases}\prec \mathbf{0}, & \mathbf{x}^{\prime} \prec \boldsymbol{\alpha}^{\prime}, \\ =\mathbf{0}, & \boldsymbol{\alpha}^{\prime} \preceq \mathbf{x}^{\prime} \preceq \boldsymbol{\beta}^{\prime}, \\ \succ \mathbf{0}, & \mathbf{x}^{\prime} \succ \boldsymbol{\beta}^{\prime},\end{cases}
$$

where $\boldsymbol{\omega}=\left(\omega_{1}, \omega_{2}, \ldots, \omega_{N}\right)^{T}$, and such a function $\boldsymbol{\omega}(\cdot)$ can be easily obtained. For example, similar to [21], let $\boldsymbol{\omega}\left(\mathbf{x}^{\prime}\right)=\mathbf{x}^{\prime}-\overline{\mathbf{x}}^{\prime}$.

Furthermore, we define

$$
\begin{aligned}
\overline{\mathbf{G}}\left(\mathbf{x}^{\prime}(t), \mathbf{x}^{\prime \prime}(t), \mathbf{x}\left(\xi_{1}\right), \ldots, \mathbf{x}\left(\xi_{m-2}\right)\right) \\
\quad=\delta\left(\boldsymbol{\alpha}^{\prime}(t), \mathbf{x}^{\prime}(t)+\mathbf{A}-\mathbf{G}\left(\mathbf{x}^{\prime}(t), \mathbf{x}^{\prime \prime}(t), \mathbf{x}\left(\xi_{1}\right), \ldots, \mathbf{x}\left(\xi_{m-2}\right)\right), \boldsymbol{\beta}^{\prime}(t)\right), \\
\overline{\mathbf{H}}\left(\mathbf{x}^{\prime}(t), \mathbf{x}^{\prime \prime}(t), \mathbf{x}\left(\eta_{1}\right), \ldots, \mathbf{x}\left(\eta_{n-2}\right)\right) \\
\quad=\delta\left(\boldsymbol{\alpha}^{\prime}(t), \mathbf{x}^{\prime}(t)+\mathbf{B}-\mathbf{H}\left(\mathbf{x}^{\prime}(t), \mathbf{x}^{\prime \prime}(t), \mathbf{x}\left(\eta_{1}\right), \ldots, \mathbf{x}\left(\eta_{n-2}\right)\right), \boldsymbol{\beta}^{\prime}(t)\right) .
\end{aligned}
$$

Then we consider the following modified problem:

$$
\left\{\begin{array}{l}
\mathbf{x}^{\prime \prime \prime}(t)+\overline{\mathbf{F}}\left(t, \mathbf{x}(t), \mathbf{x}^{\prime}(t), \mathbf{x}^{\prime \prime}(t)\right)=\mathbf{0} \\
\mathbf{x}(0)=\mathbf{0} \\
\mathbf{x}^{\prime}(0)=\overline{\mathbf{G}}\left(\mathbf{x}^{\prime}(0), \mathbf{x}^{\prime \prime}(0), \mathbf{x}\left(\xi_{1}\right), \ldots, \mathbf{x}\left(\xi_{m-2}\right)\right) \\
\mathbf{x}^{\prime}(1)=\overline{\mathbf{H}}\left(\mathbf{x}^{\prime}(1), \mathbf{x}^{\prime \prime}(1), \mathbf{x}\left(\eta_{1}\right), \ldots, \mathbf{x}\left(\eta_{n-2}\right)\right)
\end{array}\right.
$$

\section{Lemma 3.1 Assume that}

(i) $(\boldsymbol{\alpha}(t), \boldsymbol{\beta}(t))$ is a lower solution-upper solution pair of BVP (1.3), (1.4), such that

$$
\boldsymbol{\alpha}_{i}^{\prime}(t) \leq \boldsymbol{\beta}_{i}^{\prime}(t), \quad 0 \leq t \leq 1, i=1,2, \ldots, N
$$

(ii) For $(t, \mathbf{x}, \mathbf{y}, \mathbf{z}) \in[0,1] \times R^{3 N}, \mathbf{F}(t, \mathbf{x}, \mathbf{y}, \mathbf{z}) \in C\left([0,1] \times R^{3 N}, R^{N}\right)$ is continuous and increasing with respect to $\mathbf{x}$, and $\mathbf{F}(t, \mathbf{x}, \mathbf{y}, \mathbf{z})$ satisfies Nagumo condition with respect to $\mathbf{z}$.

Then BVP (3.10) has a solution $\mathbf{x}(t)=\left(x_{1}(t), \ldots, x_{N}(t)\right)^{T} \in C^{3}\left([0,1], R^{N}\right)$, such that

$$
\begin{aligned}
& \alpha_{i}(t) \leq x_{i}(t) \leq \beta_{i}(t), \quad \alpha_{i}^{\prime}(t) \leq x_{i}^{\prime}(t) \leq \beta_{i}^{\prime}(t), \quad 0 \leq t \leq 1 ; \\
& \left|x_{i}^{\prime \prime}(t)\right| \leq D_{i}, \quad i=1,2, \ldots, N,
\end{aligned}
$$

where $\mathbf{D}=\left(D_{1}, \ldots, D_{N}\right)^{T} \in R^{N}$ is concerned by (3.5), (3.6). 
Proof First, we prove that (3.10) has a solution $\mathbf{x}(t)=\left(x_{1}(t), \ldots, x_{N}(t)\right)^{T} \in C^{3}\left([0,1], R^{N}\right)$. We consider the following differential systems:

$$
\left\{\begin{array}{l}
\mathbf{x}^{\prime \prime \prime}(t)=-\lambda \overline{\mathbf{F}}\left(t, \mathbf{x}(t), \mathbf{x}^{\prime}(t), \mathbf{x}^{\prime \prime}(t)\right)=: \boldsymbol{\Psi}(t), \\
\mathbf{x}(0)=\mathbf{0} \\
\mathbf{x}^{\prime}(0)=\lambda \overline{\mathbf{G}}\left(\mathbf{x}^{\prime}(0), \mathbf{x}^{\prime \prime}(0), \mathbf{x}\left(\xi_{1}\right), \ldots, \mathbf{x}\left(\xi_{m-2}\right)\right)=: \boldsymbol{\Psi}_{*}(0), \\
\mathbf{x}^{\prime}(1)=\lambda \overline{\mathbf{H}}\left(\mathbf{x}^{\prime}(1), \mathbf{x}^{\prime \prime}(1), \mathbf{x}\left(\eta_{1}\right), \ldots, \mathbf{x}\left(\eta_{n-2}\right)\right)=: \boldsymbol{\Psi}_{*}(1)
\end{array}\right.
$$

where $\lambda \in[0,1]$. From the representations of $\overline{\mathbf{F}}, \overline{\mathbf{G}}, \overline{\mathbf{H}}$, we see that $\mathbf{x}^{\prime \prime \prime}(t), \mathbf{x}^{\prime}(0)$ and $\mathbf{x}^{\prime}(1)$ in (3.13) are bounded. Thus $\mathbf{x}^{\prime \prime}(t), \mathbf{x}^{\prime}(t), \mathbf{x}(t), 0 \leq t \leq 1$ are bounded. Consider the set

$$
\Omega=\left\{\mathbf{x}(t) \in R^{N}:\left\|\mathbf{x}^{(s)}(t)\right\|<K, s=0,1,2, K\right. \text { is some sufficiently }
$$

large positive constant, $t \in[0,1]\}$.

Then $\Omega$ is a bounded open set. BVP (3.13) can be equal to the following integral equation:

$$
\mathbf{x}(t)=\mathbf{c}_{1}+\mathbf{c}_{2} t+\mathbf{c}_{3} t^{2}+\int_{0}^{t} \int_{0}^{t_{2}} \int_{1}^{t_{1}} \boldsymbol{\Psi}(s) d s d t_{1} d t_{2}=: T_{\lambda} \mathbf{x},
$$

where $T_{\lambda}$ is an integral operator with a parameter $\lambda$, and $\left(\mathbf{c}_{1}, \mathbf{c}_{2}, \mathbf{c}_{3}\right) \in R^{N} \times R^{N} \times R^{N}$ is determined as

$$
\left\{\begin{array}{l}
\mathbf{c}_{1}=\mathbf{0}, \\
\mathbf{c}_{2}=\boldsymbol{\Psi}_{*}(0), \\
\mathbf{c}_{2}+2 \mathbf{c}_{3}=\boldsymbol{\Psi}_{*}(1)-\int_{0}^{1} \int_{1}^{t_{1}} \boldsymbol{\Psi}(s) d s d t_{1} .
\end{array}\right.
$$

Let $\mathbf{W}(\lambda, \mathbf{x})=\left(I-T_{\lambda}\right)(\mathbf{x})$, thus $\mathbf{W}:[0,1] \times \bar{\Omega} \rightarrow R^{N}$ is continuous, where $I$ is identical mapping. Let $\mathbf{w}_{\lambda}(\mathbf{x})=\mathbf{W}(\lambda, \mathbf{x}), \forall \mathbf{x} \in \partial \Omega$, due to $K$ is sufficiently large, we have

$$
\left\|\mathbf{w}_{\lambda}(\mathbf{x})\right\|=\left\|\mathbf{x}-T_{\lambda} \mathbf{x}\right\| \geq\|\mathbf{x}\|-\left\|T_{\lambda} \mathbf{x}\right\|=K-\left\|T_{\lambda} \mathbf{x}\right\|>0, \quad \forall \lambda \in[0,1] .
$$

Thus, $\mathbf{0} \notin \mathbf{w}_{\lambda}(\partial \Omega)$. According to the homotopy invariance theorem of topological degree, $\operatorname{deg}\left(\mathbf{w}_{\lambda}, \Omega, \mathbf{0}\right)$ keeps constant, in particular, $\operatorname{deg}\left(\mathbf{w}_{1}, \Omega, \mathbf{0}\right)=\operatorname{deg}\left(\mathbf{w}_{0}, \Omega, \mathbf{0}\right)$. Noticing that $\mathbf{0} \in$ $\Omega$, by the normality of topological degree, we have

$$
\operatorname{deg}\left(\mathbf{w}_{0}(\mathbf{x}), \Omega, \mathbf{0}\right)=\operatorname{deg}\left(\mathbf{x}-T_{0} \mathbf{x}, \Omega, \mathbf{0}\right)=\operatorname{deg}(\mathbf{x}, \Omega, \mathbf{0})=1
$$

and

$$
\operatorname{deg}\left(\mathbf{w}_{1}(\mathbf{x}), \Omega, \mathbf{0}\right)=\operatorname{deg}\left(\mathbf{x}-T_{1} \mathbf{x}, \Omega, \mathbf{0}\right)=\operatorname{deg}\left(\mathbf{x}-T_{0} \mathbf{x}, \Omega, \mathbf{0}\right)=1 .
$$

Hence, by the solvability theorem of topological degree, $\mathbf{w}_{1}(\mathbf{x})=\mathbf{0}$ has at least one solution. That is to say, $\mathbf{x}(t)=T_{1} \mathbf{x}$ has solutions $\mathbf{x}(t)$, it is clear that there exists some $\mathbf{x}(t) \in C^{3}\left([0,1], R^{N}\right)$ satisfying $(3.10)$.

Next, we prove that every solution $\mathbf{x}(t)$ of BVP (3.10) satisfies (3.11). First of all, we prove

$$
\alpha_{i}^{\prime}(t) \leq x_{i}^{\prime}(t) \leq \beta_{i}^{\prime}(t), \quad 0 \leq t \leq 1, i=1,2, \ldots, N,
$$


if $\alpha_{i}^{\prime}(t) \leq x_{i}^{\prime}(t), i=1,2, \ldots, N$, is not true, then there exist some $i \in\{1,2, \ldots, N\}$ and $\zeta \in[0,1]$, such that

$$
\max _{0 \leq t \leq 1}\left(\alpha_{i}^{\prime}(t)-x_{i}^{\prime}(t)\right)=\alpha_{i}^{\prime}(\zeta)-x_{i}^{\prime}(\zeta)>0
$$

Obviously, from the boundary conditions of BVP (3.10), we know $\zeta \neq 0,1$. Thus

$$
\begin{aligned}
& \alpha_{i}^{\prime \prime}(\zeta)-x_{i}^{\prime \prime}(\zeta)=0 \\
& \alpha_{i}^{\prime \prime \prime}(\zeta)-x_{i}^{\prime \prime \prime}(\zeta) \leq 0
\end{aligned}
$$

From conditions (i), (ii) and (2.1), (3.1)-(3.5), (3.7), (3.16), Definition 2 and the fact that $\mathbf{x}(t)$ is a solution of (3.10), we have

$$
\begin{aligned}
\alpha_{i}^{\prime \prime \prime}(\zeta)-x_{i}^{\prime \prime \prime}(\zeta) & \geq-f_{i}\left(\zeta, \mathbf{x}_{\alpha_{i}}(\zeta), \mathbf{x}_{\alpha_{i}}^{\prime}(\zeta), \mathbf{x}_{\alpha_{i}}^{\prime \prime}(\zeta)\right)+\bar{f}_{i}\left(\zeta, \mathbf{x}(\zeta), \mathbf{x}^{\prime}(\zeta), \mathbf{x}^{\prime \prime}(\zeta)\right) \\
& =-f_{i}\left(\zeta, \mathbf{x}_{\alpha_{i}}(\zeta), \mathbf{x}_{\alpha_{i}}^{\prime}(\zeta), \mathbf{x}_{\alpha_{i}}^{\prime \prime}(\zeta)\right)+f_{i}\left(\zeta, \overline{\mathbf{x}}(\zeta), \overline{\mathbf{x}}^{\prime}(\zeta), \overline{\mathbf{x}}^{\prime \prime}(\zeta)\right)-\omega_{i}\left(\mathbf{x}^{\prime}(\zeta)\right) \\
& =-f_{i}\left(\zeta, \mathbf{x}_{\alpha_{i}}(\zeta), \mathbf{x}_{\alpha_{i}}^{\prime}(\zeta), \mathbf{x}^{\prime \prime}(\zeta)\right)+f_{i}\left(\zeta, \overline{\mathbf{x}}(\zeta), \mathbf{x}_{\alpha_{i}}^{\prime}(\zeta), \mathbf{x}^{\prime \prime}(\zeta)\right)-\omega_{i}\left(\mathbf{x}^{\prime}(\zeta)\right) \\
& \geq 0-\omega_{i}\left(\mathbf{x}^{\prime}(\zeta)\right)>0,
\end{aligned}
$$

it is contradictory to (3.17), hence we obtain $\alpha_{i}^{\prime}(t) \leq x_{i}^{\prime}(t), 0 \leq t \leq 1$.

Similarly, we could prove that $x_{i}^{\prime}(t) \leq \beta_{i}^{\prime}(t), 0 \leq t \leq 1$.

Thus, (3.15) is true. According to condition (i) and Definition 4, we have $\alpha_{i}(0) \leq x_{i}(0) \leq$ $\beta_{i}(0)$, by integrating the inequalities (3.15) on $[0, t]$, we obtain

$$
\alpha_{i}(t) \leq x_{i}(t) \leq \beta_{i}(t), \quad 0 \leq t \leq 1
$$

Finally, we prove (3.12) holds. We suppose that $\left|x_{i}^{\prime \prime}(t)\right| \leq D_{i}$ is not true. Then there exists $\sigma \in[0,1]$, such that $x_{i}^{\prime \prime}(\sigma)>D_{i}$, or $x_{i}^{\prime \prime}(\sigma)<-D_{i}$. Suppose that the first case holds. From (3.5), (3.6) and $\mathbf{F}(t)$ is continuous, there exists $\varsigma \in[0,1]$ such that

$$
x_{i}^{\prime \prime}(\varsigma)=\frac{x_{i}^{\prime}(1)-x_{i}^{\prime}(0)}{1-0} \leq \beta_{i}^{\prime}(1)-\alpha_{i}^{\prime}(0) \leq 2 M_{i}<D_{i} .
$$

Because $\mathbf{x}^{\prime \prime}(t)$ is continuous and $x_{i}^{\prime \prime}(\sigma)>D_{i}$, there exists some subinterval $[a, b]($ or $[b, a]) \subset$ $[0,1]$ such that

$$
\begin{aligned}
& x_{i}^{\prime \prime}(a)=2 M_{i}, \quad x_{i}^{\prime \prime}(b)=D_{i}, \\
& 2 M_{i}<x_{i}^{\prime \prime}(t)<D_{i}, \quad \forall t \in[a, b](\text { or }[b, a]) .
\end{aligned}
$$

From condition (ii) and Definition 3, one has

$$
\left|\int_{a}^{b} \frac{x_{i}^{\prime \prime}(s) x_{i}^{\prime \prime \prime}(s)}{\Phi_{i}\left(x_{i}^{\prime \prime}(s)\right)} d s\right| \leq\left|\int_{a}^{b} x_{i}^{\prime \prime}(s) d s\right|=\left|x_{i}^{\prime}(b)-x_{i}^{\prime}(a)\right| \leq 2 M_{i} .
$$

On the other hand, from (3.5) and (3.6), we know that

$$
\left|\int_{a}^{b} \frac{x_{i}^{\prime \prime}(s) x_{i}^{\prime \prime \prime}(s)}{\Phi_{i}\left(x_{i}^{\prime \prime}(s)\right)} d s\right|=\left|\int_{2 M_{i}}^{D_{i}} \frac{s d s}{\Phi_{i}(s)}\right|=\int_{2 M_{i}}^{D_{i}} \frac{s d s}{\Phi_{i}(s)}>2 M_{i} .
$$


This inequality is contradictory to the above one. So we show that $x_{i}^{\prime \prime}(\sigma)>D_{i}$ is not true. Similarly, we can prove that $x_{i}^{\prime \prime}(\sigma)<-D_{i}$ is not true too. Therefore, (3.12) holds.

\subsection{Existence result of BVP (1.3), (1.4)}

Theorem 3.1 Assume that conditions (i), (ii) in Lemma 3.1 hold and

(iii) $\mathbf{G}\left(\mathbf{x}_{1}, \mathbf{x}_{2}, \ldots, \mathbf{x}_{m}\right)$ is continuous and decreasing with respect to $\mathbf{x}_{2}, \ldots, \mathbf{x}_{m}$;

$\mathbf{H}\left(\mathbf{y}_{1}, \mathbf{y}_{2}, \ldots, \mathbf{y}_{n}\right)$ is continuous and increasing in $\mathbf{y}_{2}$ and decreasing with respect to $\mathbf{y}_{3}, \ldots, \mathbf{y}_{n}$.

Then BVP (1.3), (1.4) has a solution $\mathbf{x}(t)=\left(x_{1}(t), \ldots, x_{N}(t)\right)^{T} \in C^{3}\left([0,1], R^{N}\right)$ satisfying inequalities (3.11) and (3.12).

Proof From (2.1), (3.1)-(3.4), (3.7) and Lemma 3.1, there exists a solution $\mathbf{x}(t)$ of the modified BVP (3.10) satisfying (1.3), (3.11) and (3.12).

Now we show the solution $\mathbf{x}(t)$ satisfying the boundary conditions (1.4). From the boundary conditions of (3.10), it is easy to get $\mathbf{x}(0)=\mathbf{0}$.

First, we prove

$$
\mathbf{G}\left(\mathbf{x}^{\prime}(0), \mathbf{x}^{\prime \prime}(0), \mathbf{x}\left(\xi_{1}\right), \mathbf{x}\left(\xi_{2}\right), \ldots, \mathbf{x}\left(\xi_{m-2}\right)\right)=\mathbf{A}
$$

Case 1. Suppose that $\boldsymbol{\alpha}^{\prime}(0) \preceq \mathbf{x}^{\prime}(0)+\mathbf{A}-\mathbf{G}\left(\mathbf{x}^{\prime}(0), \mathbf{x}^{\prime \prime}(0), \mathbf{x}\left(\xi_{1}\right), \mathbf{x}\left(\xi_{2}\right), \ldots, \mathbf{x}\left(\xi_{m-2}\right)\right) \preceq \boldsymbol{\beta}^{\prime}(0)$. By (2.1), (3.8) and (3.10), we obtain

$$
\begin{aligned}
\mathbf{x}^{\prime}(0) & =\overline{\mathbf{G}}\left(\mathbf{x}^{\prime}(0), \mathbf{x}^{\prime \prime}(0), \mathbf{x}\left(\xi_{1}\right), \mathbf{x}\left(\xi_{2}\right), \ldots, \mathbf{x}\left(\xi_{m-2}\right)\right) \\
& =\mathbf{x}^{\prime}(0)+\mathbf{A}-\mathbf{G}\left(\mathbf{x}^{\prime}(0), \mathbf{x}^{\prime \prime}(0), \mathbf{x}\left(\xi_{1}\right), \mathbf{x}\left(\xi_{2}\right), \ldots, \mathbf{x}\left(\xi_{m-2}\right)\right) .
\end{aligned}
$$

Thus (3.18) holds.

Case 2. Suppose that $\boldsymbol{\alpha}^{\prime}(0) \succ \mathbf{x}^{\prime}(0)+\mathbf{A}-\mathbf{G}\left(\mathbf{x}^{\prime}(0), \mathbf{x}^{\prime \prime}(0), \mathbf{x}\left(\xi_{1}\right), \mathbf{x}\left(\xi_{2}\right), \ldots, \mathbf{x}\left(\xi_{m-2}\right)\right)$. By (2.1), (3.8) and (3.10), we obtain

$$
\mathbf{x}^{\prime}(0)=\overline{\mathbf{G}}\left(\mathbf{x}^{\prime}(0), \mathbf{x}^{\prime \prime}(0), \mathbf{x}\left(\xi_{1}\right), \mathbf{x}\left(\xi_{2}\right), \ldots, \mathbf{x}\left(\xi_{m-2}\right)\right)=\boldsymbol{\alpha}^{\prime}(0) .
$$

Then

$$
\mathbf{G}\left(\mathbf{x}^{\prime}(0), \mathbf{x}^{\prime \prime}(0), \mathbf{x}\left(\xi_{1}\right), \mathbf{x}\left(\xi_{2}\right), \ldots, \mathbf{x}\left(\xi_{m-2}\right)\right) \succ \mathbf{A} .
$$

According to (3.11), (3.19) and condition (iii), we know

$$
\mathbf{G}\left(\boldsymbol{\alpha}^{\prime}(0), \boldsymbol{\alpha}^{\prime \prime}(0), \boldsymbol{\alpha}\left(\xi_{1}\right), \ldots, \boldsymbol{\alpha}\left(\xi_{m-2}\right)\right) \succeq \mathbf{G}\left(\mathbf{x}^{\prime}(0), \mathbf{x}^{\prime \prime}(0), \mathbf{x}\left(\xi_{1}\right), \mathbf{x}\left(\xi_{2}\right), \ldots, \mathbf{x}\left(\xi_{m-2}\right)\right) .
$$

Therefore,

$$
\mathbf{G}\left(\boldsymbol{\alpha}^{\prime}(0), \boldsymbol{\alpha}^{\prime \prime}(0), \boldsymbol{\alpha}\left(\xi_{1}\right), \ldots, \boldsymbol{\alpha}\left(\xi_{m-2}\right)\right) \succ \mathbf{A} .
$$

From condition (i), it is easy to see that (3.21) is contradictory to Definition 4. Therefore, (3.20) is not true. 
Case 3. Suppose that $\mathbf{x}^{\prime}(0)+\mathbf{A}-\mathbf{G}\left(\mathbf{x}^{\prime}(0), \mathbf{x}^{\prime \prime}(0), \mathbf{x}\left(\xi_{1}\right), \mathbf{x}\left(\xi_{2}\right), \ldots, \mathbf{x}\left(\xi_{m-2}\right)\right) \succ \boldsymbol{\beta}^{\prime}(0)$. By (2.1), (3.8) and (3.10), we obtain

$$
\begin{aligned}
\mathbf{x}^{\prime}(0) & =\overline{\mathbf{G}}\left(\mathbf{x}^{\prime}(0), \mathbf{x}^{\prime \prime}(0), \mathbf{x}\left(\xi_{1}\right), \mathbf{x}\left(\xi_{2}\right), \ldots, \mathbf{x}\left(\xi_{m-2}\right)\right) \\
& =\boldsymbol{\beta}^{\prime}(0) .
\end{aligned}
$$

So

$$
\mathbf{G}\left(\mathbf{x}^{\prime}(0), \mathbf{x}^{\prime \prime}(0), \mathbf{x}\left(\xi_{1}\right), \mathbf{x}\left(\xi_{2}\right), \ldots, \mathbf{x}\left(\xi_{m-2}\right)\right) \prec \mathbf{A} .
$$

In view of (3.11), (3.22) and condition (iii), we know

$$
\mathbf{G}\left(\boldsymbol{\beta}^{\prime}(0), \boldsymbol{\beta}^{\prime \prime}(0), \boldsymbol{\beta}\left(\xi_{1}\right), \ldots, \boldsymbol{\beta}\left(\xi_{m-2}\right)\right) \preceq \mathbf{G}\left(\mathbf{x}^{\prime}(0), \mathbf{x}^{\prime \prime}(0), \mathbf{x}\left(\xi_{1}\right), \mathbf{x}\left(\xi_{2}\right), \ldots, \mathbf{x}\left(\xi_{m-2}\right)\right),
$$

thus,

$$
\mathbf{G}\left(\boldsymbol{\beta}^{\prime}(0), \boldsymbol{\beta}^{\prime \prime}(0), \boldsymbol{\beta}\left(\xi_{1}\right), \ldots, \boldsymbol{\beta}\left(\xi_{m-2}\right)\right) \prec \mathbf{A} .
$$

By condition (i), it is easy to see that (3.24) is also contradictory to Definition 4. Therefore, (3.23) is not true too. Thus, we show that (3.18) holds.

Similar to the above argument, we could prove that

$$
\mathbf{H}\left(\mathbf{x}^{\prime}(1), \mathbf{x}^{\prime \prime}(1), \mathbf{x}\left(\eta_{1}\right), \mathbf{x}\left(\eta_{2}\right), \ldots, \mathbf{x}\left(\eta_{n-2}\right)\right)=\mathbf{B}
$$

Thus $\mathbf{x}(t)$ is a solution of BVP (1.3), (1.4) and satisfies (3.11), (3.12).

\subsection{Existence result of SPBVP (1.1), (1.2)}

\section{Theorem 3.2 Assume that}

(i) The reduced problem of SPBVP (1.1), (1.2)

$$
\left\{\begin{array}{l}
\mathbf{F}\left(t, \mathbf{x}, \mathbf{x}^{\prime}, \mathbf{x}^{\prime \prime}, 0\right)=\mathbf{0}, \\
\mathbf{x}(0)=\mathbf{0}, \quad \mathbf{G}\left(\mathbf{x}^{\prime}(0), \mathbf{x}^{\prime \prime}(0), \mathbf{x}\left(\xi_{1}\right), \mathbf{x}\left(\xi_{2}\right), \ldots, \mathbf{x}\left(\xi_{m-2}\right)\right)=\mathbf{A},
\end{array}\right.
$$

has a reduced solution $\mathbf{v}(t)=\left(v_{1}(t), \ldots, v_{N}(t)\right)^{T} \in C^{3}\left([0,1], R^{N}\right)$. For $i=1,2, \ldots, N, v_{i}(t)$ satisfies

$$
\begin{aligned}
& f_{i}\left(t, \mathbf{x}_{v_{i}}(t, \varepsilon), \mathbf{x}_{v_{i}}^{\prime}(t, \varepsilon), \mathbf{x}_{v_{i}}^{\prime \prime}(t, \varepsilon), 0\right)=f_{i}\left(t, \mathbf{x}_{v_{i}}(t, 0), \mathbf{x}_{v_{i}}^{\prime}(t, 0), \mathbf{x}_{v_{i}}^{\prime \prime}(t, 0), 0\right)=0, \\
& v_{i}(0)=0, \quad g_{i}\left(\mathbf{v}^{\prime}(0), \mathbf{v}^{\prime \prime}(0), \mathbf{v}\left(\xi_{1}\right), \mathbf{v}\left(\xi_{2}\right), \ldots, \mathbf{v}\left(\xi_{m-2}\right)\right)=A_{i} ;
\end{aligned}
$$

(ii) Let $\varepsilon_{0}$ be a sufficiently small constant, $f_{i}\left(t, \mathbf{x}, \mathbf{x}^{\prime}, \mathbf{x}^{\prime \prime}, \varepsilon\right), i=1,2, \ldots, N$, is continuously differentiable and satisfies Nagumo condition on $[0,1] \times R^{3 N} \times\left[0, \varepsilon_{0}\right]$ and there exist some positive constants $l_{i}, r_{i}, c_{i}, i=1,2, \ldots, N$, such that

$$
\begin{aligned}
& 0<f_{i x_{i}}(t, \mathbf{x}, \mathbf{y}, \mathbf{z}, \varepsilon) \leq l_{i}, \quad f_{i y_{i}}(t, \mathbf{x}, \mathbf{y}, \mathbf{z}, \varepsilon) \leq-r_{i}<0 \\
& f_{i z_{i}}(t, \mathbf{x}, \mathbf{y}, \mathbf{z}, \varepsilon) \leq 0, \quad\left|f_{i \varepsilon}(t, \mathbf{x}, \mathbf{y}, \mathbf{z}, \varepsilon)\right| \leq c_{i}
\end{aligned}
$$

where $f_{i x_{i}}=\frac{\partial f_{i}(t, \mathbf{x}, \mathbf{y}, \mathbf{z})}{\partial x_{i}}$, the others are defined analogously. 
(iii) $\mathbf{G}\left(\mathbf{x}_{1}, \ldots, \mathbf{x}_{m}\right)$ is continuous and increasing in $\mathbf{x}_{1}$ and decreasing with respect to $\mathbf{x}_{2}, \ldots, \mathbf{x}_{m} ; \mathbf{H}\left(\mathbf{y}_{1}, \ldots, \mathbf{y}_{n}\right)$ is continuous and increasing with respect to $\mathbf{y}_{1}, \mathbf{y}_{2}$ and decreasing with respect to $\mathbf{y}_{3}, \ldots, \mathbf{y}_{n}$. And there exist some vectors $\mathbf{M}_{s}=\left(M_{s 1}, M_{s 2}, \ldots, M_{s N}\right)^{T} \succ \mathbf{0}, s=1,2, \ldots, 6$, such that $\mathbf{v}^{\prime \prime}(0) \prec-\mathbf{M}_{1}, \mathbf{v}^{\prime \prime}(1) \succ \mathbf{M}_{2}$, and

$$
\begin{aligned}
& g_{i}\left(\mathbf{v}^{\prime}(0), \mathbf{M}_{1}, \mathbf{M}_{5}, \ldots, \mathbf{M}_{5}\right) \leq A_{i} \leq g_{i}\left(\mathbf{v}^{\prime}(0),-\mathbf{M}_{1}, \mathbf{M}_{3}, \ldots, \mathbf{M}_{3}\right) \\
& h_{i}\left(\mathbf{v}^{\prime}(1),-\mathbf{M}_{2}, \mathbf{M}_{6}, \ldots, \mathbf{M}_{6}\right) \leq B_{i} \leq h_{i}\left(\mathbf{v}^{\prime}(1), \mathbf{M}_{2}, \mathbf{M}_{4}, \ldots, \mathbf{M}_{4}\right)
\end{aligned}
$$

Then SPBVP (1.1), (1.2) has a solution $\mathbf{x}(t, \varepsilon)=\left(x_{1}(t, \varepsilon), \ldots, x_{N}(t, \varepsilon)\right)^{T}$ such that

$$
\left|x_{i}(t, \varepsilon)-v_{i}(t)\right| \leq T_{1 i} e^{\lambda_{1 i} t}+T_{2 i} e^{\lambda_{2 i}(t-1)}+T_{3 i} \varepsilon, \quad i=1,2, \ldots, N
$$

where $T_{\kappa}=\operatorname{diag}\left(T_{\kappa 1}, T_{\kappa 2}, \ldots, T_{\kappa N}\right), T_{\kappa i}(\kappa=1,2,3, i=1,2, \ldots, N)$ are positive numbers. $\varepsilon$ is sufficiently small, $\lambda_{1 i}, \lambda_{2 i}$ are two roots of equation $\varepsilon \lambda^{3}-r_{i} \lambda+l_{i}=0$, such that

$$
-2 \sqrt{\frac{r_{i}}{\varepsilon}}<\lambda_{1 i}<-\sqrt{\frac{r_{i}}{\varepsilon}}, \quad \frac{1}{2} \sqrt{\frac{r_{i}}{\varepsilon}}<\lambda_{2 i}<\sqrt{\frac{r_{i}}{\varepsilon}} .
$$

Proof From condition (i), there exists a positive constant vector $\mathbf{M}^{*}=\left(M_{1}^{*}, M_{2}^{*}, \ldots, M_{N}^{*}\right)^{T}$, such that $\left|v_{i}^{\prime \prime \prime}(t)\right| \leq M_{i}^{*}, i=1,2, \ldots, N$, since $\mathbf{v}(t) \in C^{3}\left([0,1], R^{N}\right)$. Then the equation $\varepsilon \lambda^{3}-$ $r_{i} \lambda+l_{i}=0$ has three different real roots $\lambda_{1 i}, \lambda_{2 i}$, and $\lambda_{3 i}$, since

$$
\frac{1}{4}\left(\frac{l_{i}}{\varepsilon}\right)^{2}+\frac{1}{27}\left(-\frac{r_{i}}{\varepsilon}\right)^{3}=\frac{1}{\varepsilon^{2}}\left(\frac{l_{i}^{2}}{4}-\frac{r_{i}^{3}}{27 \varepsilon}\right)<0
$$

Furthermore, for $i=1,2, \ldots, N$, the estimates of $\lambda_{1 i}, \lambda_{2 i}$ are given in (3.29) and have the estimate of $\lambda_{3 i}$ satisfies

$$
\frac{l_{i}}{r_{i}}<\lambda_{3 i}<\frac{l_{i}+r_{i}}{r_{i}}
$$

To construct the upper and lower solutions, we define

$$
\gamma_{i}(t, \varepsilon)=\varepsilon^{\frac{1}{2}}\left[\frac{d_{1 i}}{\lambda_{1 i}} e^{\lambda_{1 i} t}+\frac{d_{2 i}}{\lambda_{2 i}} e^{\lambda_{2 i}(t-1)}\right]+\frac{d_{3 i}}{\lambda_{3 i}}\left[2 e^{\lambda_{3 i} t}-1\right],
$$

where

$$
d_{1 i}=-\frac{M_{1 i}+\left|v_{i}^{\prime \prime}(0)\right|+1}{\lambda_{1 i} \varepsilon^{\frac{1}{4}}}, \quad d_{2 i}=\frac{M_{2 i}+\left|v_{i}^{\prime \prime}(1)\right|+1}{\lambda_{2 i} \varepsilon^{\frac{1}{2}}}, \quad d_{3 i}=\frac{\lambda_{3 i}\left(c_{i}+M_{i}^{*}+1\right)}{l_{i}} \varepsilon^{\frac{1}{5}} .
$$

Then we have

$$
\begin{aligned}
& \gamma_{i}^{\prime}(t, \varepsilon)=\varepsilon^{\frac{1}{2}}\left[d_{1 i} e^{\lambda_{1 i} t}+d_{2 i} e^{\lambda_{2 i}(t-1)}\right]+2 d_{3 i} e^{\lambda_{3 i} t}, \\
& \gamma_{i}^{\prime \prime}(t, \varepsilon)=\varepsilon^{\frac{1}{2}}\left[d_{1 i} \lambda_{1 i} e^{\lambda_{1 i} t}+d_{2 i} \lambda_{2 i} e^{\lambda_{2 i}(t-1)}\right]+2 d_{3 i} \lambda_{3 i} e^{\lambda_{3 i} t} \\
& \gamma_{i}^{\prime \prime \prime}(t, \varepsilon)=\varepsilon^{\frac{1}{2}}\left[d_{1 i} \lambda_{1 i}^{2} e^{\lambda_{1 i} t}+d_{2 i} \lambda_{2 i}^{2} e^{\lambda_{2 i}(t-1)}\right]+2 d_{3 i} \lambda_{3 i}^{2} e^{\lambda_{3 i} t}
\end{aligned}
$$


In view of $d_{1 i}>0, d_{2 i}>0, d_{3 i}>0$, we obtain

$$
\gamma_{i}^{\prime}(t, \varepsilon)>0, \quad \gamma_{i}^{\prime \prime \prime}(t, \varepsilon)>0, \quad 0 \leq t \leq 1, \varepsilon>0 .
$$

For sufficiently small $\varepsilon>0$, we have

$$
\begin{aligned}
\gamma_{i}(0, \varepsilon) & =\varepsilon^{\frac{1}{2}}\left(\frac{d_{1 i}}{\lambda_{1 i}}+\frac{d_{2 i}}{\lambda_{2 i}} e^{-\lambda_{2 i}}\right)+\frac{d_{3 i}}{\lambda_{3 i}} \\
& =-\frac{M_{1 i}+\left|v_{i}^{\prime \prime}(0)\right|+1}{\lambda_{1 i}^{2}} \varepsilon^{\frac{1}{4}}+\frac{M_{2 i}+\left|v_{i}^{\prime \prime}(1)\right|+1}{\lambda_{2 i}^{2}} e^{-\lambda_{2 i}}+\frac{c_{i}+M_{i}^{*}+1}{l_{i}} \varepsilon^{\frac{1}{5}} \\
& >-\frac{M_{1 i}+\left|v_{i}^{\prime \prime}(0)\right|+1}{r_{i}} \varepsilon^{\frac{5}{4}}+\frac{M_{2 i}+\left|v_{i}^{\prime \prime}(1)\right|+1}{r_{i}} \varepsilon e^{-\sqrt{\frac{r_{i}}{\varepsilon}}+\frac{c_{i}+M_{i}^{*}+1}{l_{i}} \varepsilon^{\frac{1}{5}}} \\
& >0
\end{aligned}
$$

since $\gamma_{i}^{\prime}(s, \varepsilon)>0$, we have $\gamma_{i}(t, \varepsilon)=\gamma_{i}(0, \varepsilon)+\int_{0}^{t} \gamma_{i}^{\prime}(s, \varepsilon) d s>0$, for $0 \leq t \leq 1$.

Similarly, we obtain

$$
\begin{aligned}
\gamma_{i}^{\prime \prime}(0, \varepsilon) & =\varepsilon^{\frac{1}{2}}\left(d_{1 i} \lambda_{1 i}+d_{2 i} \lambda_{2 i} e^{-\lambda_{2 i}}\right)+2 d_{3 i} \lambda_{3 i} \\
& >-\left(M_{1 i}+\left|v_{i}^{\prime \prime}(0)\right|+1\right) \varepsilon^{\frac{1}{4}}+\left(M_{2 i}+\left|v_{i}^{\prime \prime}(1)\right|+1\right) \varepsilon^{-\sqrt{\frac{r_{i}}{\varepsilon}}}+\frac{2 l_{i}\left(c_{i}+M_{i}^{*}+1\right)}{r_{i}^{2}} \varepsilon^{\frac{1}{5}} \\
& >0 .
\end{aligned}
$$

Thus, $\gamma_{i}^{\prime \prime}(t, \varepsilon)=\gamma_{i}^{\prime \prime}(0, \varepsilon)+\int_{0}^{t} \gamma_{i}^{\prime \prime \prime}(s, \varepsilon) d s>0$, for $0 \leq t \leq 1$, since $\gamma_{i}^{\prime \prime \prime}(s, \varepsilon)>0$.

Define functions $\boldsymbol{\beta}(t, \varepsilon), \boldsymbol{\alpha}(t, \varepsilon)$ as

$$
\boldsymbol{\beta}(t, \varepsilon)=\mathbf{v}(t)+\boldsymbol{\gamma}(t, \varepsilon), \quad \boldsymbol{\alpha}(t, \varepsilon)=\mathbf{v}(t)-\boldsymbol{\gamma}(t, \varepsilon),
$$

where

$$
\boldsymbol{\gamma}(t, \varepsilon)=\left(\gamma_{1}(t, \varepsilon), \gamma_{2}(t, \varepsilon), \ldots, \gamma_{N}(t, \varepsilon)\right)^{T}
$$

Hence

$$
\beta_{i}(t, \varepsilon)=v_{i}(t)+\gamma_{i}(t, \varepsilon), \quad \alpha_{i}(t, \varepsilon)=v_{i}(t)-\gamma_{i}(t, \varepsilon), \quad i=1,2, \ldots, N
$$

For $(t, \varepsilon) \in[0,1] \times\left[0, \varepsilon_{0}\right]$, we have

$$
\begin{array}{ll}
\alpha_{i}(t, \varepsilon) \leq \beta_{i}(t, \varepsilon), & \alpha_{i}^{\prime}(t, \varepsilon) \leq \beta_{i}^{\prime}(t, \varepsilon), \\
\alpha_{i}^{\prime \prime}(t, \varepsilon) \leq \beta_{i}^{\prime \prime}(t, \varepsilon), & \alpha_{i}(0, \varepsilon) \leq 0 \leq \beta_{i}(0, \varepsilon),
\end{array}
$$

and

$$
\begin{aligned}
\varepsilon \beta_{i}^{\prime \prime \prime}( & t, \varepsilon)+f_{i}\left(t, \mathbf{x}_{\beta_{i}}(t, \varepsilon), \mathbf{x}_{\beta_{i}}^{\prime}(t, \varepsilon), \mathbf{x}_{\beta_{i}}^{\prime \prime}(t, \varepsilon), \varepsilon\right) \\
= & \varepsilon \beta_{i}^{\prime \prime \prime}(t, \varepsilon)+f_{i}\left(t, \mathbf{x}_{\beta_{i}}(t, \varepsilon), \mathbf{x}_{\beta_{i}}^{\prime}(t, \varepsilon), \mathbf{x}_{\beta_{i}}^{\prime \prime}(t, \varepsilon), \varepsilon\right)-f_{i}\left(t, \mathbf{x}_{\beta_{i}}(t, \varepsilon), \mathbf{x}_{\beta_{i}}^{\prime}(t, \varepsilon), \mathbf{x}_{v_{i}}^{\prime \prime}(t, \varepsilon), \varepsilon\right) \\
& +f_{i}\left(t, \mathbf{x}_{\beta_{i}}(t, \varepsilon), \mathbf{x}_{\beta_{i}}^{\prime}(t, \varepsilon), \mathbf{x}_{v_{i}}^{\prime \prime}(t, \varepsilon), \varepsilon\right)-f_{i}\left(t, \mathbf{x}_{\beta_{i}}(t, \varepsilon), \mathbf{x}_{v_{i}}^{\prime}(t, \varepsilon), \mathbf{x}_{v_{i}}^{\prime \prime}(t, \varepsilon), \varepsilon\right)
\end{aligned}
$$




$$
\begin{aligned}
& +f_{i}\left(t, \mathbf{x}_{\beta_{i}}(t, \varepsilon), \mathbf{x}_{v_{i}}^{\prime}(t, \varepsilon), \mathbf{x}_{v_{i}}^{\prime \prime}(t, \varepsilon), \varepsilon\right)-f_{i}\left(t, \mathbf{x}_{v_{i}}(t, \varepsilon), \mathbf{x}_{v_{i}}^{\prime}(t, \varepsilon), \mathbf{x}_{v_{i}}^{\prime \prime}(t, \varepsilon), \varepsilon\right) \\
& +f_{i}\left(t, \mathbf{x}_{v_{i}}(t, \varepsilon), \mathbf{x}_{v_{i}}^{\prime}(t, \varepsilon), \mathbf{x}_{v_{i}}^{\prime \prime}(t, \varepsilon), \varepsilon\right)-f_{i}\left(t, \mathbf{x}_{v_{i}}(t, \varepsilon), \mathbf{x}_{v_{i}}^{\prime}(t, \varepsilon), \mathbf{x}_{v_{i}}^{\prime \prime}(t, \varepsilon), 0\right) \\
& +f_{i}\left(t, \mathbf{x}_{v_{i}}(t, \varepsilon), \mathbf{x}_{v_{i}}^{\prime}(t, \varepsilon), \mathbf{x}_{v_{i}}^{\prime \prime}(t, \varepsilon), 0\right) \\
& =\varepsilon \beta_{i}^{\prime \prime \prime}(t, \varepsilon)+\int_{0}^{1} f_{i z_{i}}\left(t, \mathbf{x}_{\beta_{i}}(t, \varepsilon), \mathbf{x}_{\beta_{i}}^{\prime}(t, \varepsilon), \mathbf{x}_{v_{i}+\theta\left(\beta_{i}-v_{i}\right)}^{\prime \prime}(t, \varepsilon), \varepsilon\right) d \theta \cdot \gamma_{i}^{\prime \prime}(t, \varepsilon) \\
& +\int_{0}^{1} f_{i y_{i}}\left(t, \mathbf{x}_{\beta_{i}}(t, \varepsilon), \mathbf{x}_{v_{i}+\theta\left(\beta_{i}-v_{i}\right)}^{\prime}(t, \varepsilon), \mathbf{x}_{v_{i}}^{\prime \prime}(t, \varepsilon), \varepsilon\right) d \theta \cdot \gamma_{i}^{\prime}(t, \varepsilon) \\
& +\int_{0}^{1} f_{i x_{i}}\left(t, \mathbf{x}_{v_{i}+\theta\left(\beta_{i}-v_{i}\right)}(t, \varepsilon), \mathbf{x}_{v_{i}}^{\prime}(t, \varepsilon), \mathbf{x}_{v_{i}}^{\prime \prime}(t, \varepsilon), \varepsilon\right) d \theta \cdot \gamma_{i}(t, \varepsilon) \\
& +\int_{0}^{1} f_{i \varepsilon}\left(t, \mathbf{x}_{v_{i}}(t, \varepsilon), \mathbf{x}_{v_{i}}^{\prime}(t, \varepsilon), \mathbf{x}_{v_{i}}^{\prime \prime}(t, \varepsilon), \theta \varepsilon\right) d \theta \cdot \varepsilon \\
& \leq \varepsilon\left(v_{i}^{\prime \prime \prime}(t)+\gamma_{i}^{\prime \prime \prime}(t, \varepsilon)\right)-r_{i} \gamma_{i}^{\prime}(t, \varepsilon)+l_{i} \gamma_{i}(t, \varepsilon)+c_{i} \varepsilon \\
& \leq \varepsilon\left(c_{i}+M_{i}^{*}\right)+\frac{\varepsilon^{\frac{1}{2}} d_{1 i}}{\lambda_{1 i}} e^{\lambda_{1 i} t}\left(\varepsilon \lambda_{1 i}^{3}-r_{i} \lambda_{1 i}+l_{i}\right)+\frac{\varepsilon^{\frac{1}{2}} d_{2 i}}{\lambda_{2 i}} e^{\lambda_{2 i}(t-1)}\left(\varepsilon \lambda_{2 i}^{3}-r_{i} \lambda_{2 i}+l_{i}\right) \\
& +\frac{2 d_{3 i}}{\lambda_{3 i}} e^{\lambda_{3 i} t}\left(\varepsilon \lambda_{3 i}^{3}-r_{i} \lambda_{3 i}+l_{i}\right)-\frac{l_{i} d_{3 i}}{\lambda_{3 i}} \\
& =\varepsilon\left(c_{i}+M_{i}^{*}\right)-\frac{l_{i} d_{3 i}}{\lambda_{3 i}} \\
& =-\varepsilon^{\frac{1}{5}}\left[\left(1+c_{i}+M_{i}^{*}\right)-\left(c_{i}+M_{i}^{*}\right) \varepsilon^{\frac{4}{5}}\right]<0,
\end{aligned}
$$

i.e.

$$
\varepsilon \beta_{i}^{\prime \prime \prime}(t, \varepsilon)+f_{i}\left(t, \mathbf{x}_{\beta_{i}}(t, \varepsilon), \mathbf{x}_{\beta_{i}}^{\prime}(t, \varepsilon), \mathbf{x}_{\beta_{i}}^{\prime \prime}(t, \varepsilon), \varepsilon\right) \leq 0
$$

Similarly, from the expression of $\beta_{i}^{\prime}(t, \varepsilon)$, we obtain $\beta_{i}^{\prime}(0, \varepsilon)=v_{i}^{\prime}(0)+\gamma_{i}^{\prime}(0, \varepsilon) \geq v_{i}^{\prime}(0)$, and $\beta_{i}^{\prime}(1, \varepsilon) \geq v_{i}^{\prime}(1)$. From condition (iii), there exists $\varepsilon_{i 1}>0$, for $0<\varepsilon \leq \varepsilon_{i 1}$, one has $\beta_{i}^{\prime \prime}(0, \varepsilon)<$ $-M_{1 i}$, since $\gamma_{i}^{\prime \prime}(0, \varepsilon)>0$ is sufficient small. Furthermore, there exists $\varepsilon_{i 2}>0$, for $0<\varepsilon \leq \varepsilon_{i 2}$, we have $\beta_{i}^{\prime \prime}(1, \varepsilon) \geq M_{2 i}$. Then there exists $\widetilde{\varepsilon}_{i k}>0$, for $0<\varepsilon \leq \widetilde{\varepsilon}_{i k}(k=1,2, \ldots, m-2)$, we have

$$
\begin{aligned}
\beta_{i}\left(\xi_{k}, \varepsilon\right)= & v_{i}\left(\xi_{k}\right)+\varepsilon^{\frac{1}{2}}\left[\frac{d_{1 i}}{\lambda_{1 i}} e^{\lambda_{11} \xi_{k}}+\frac{d_{2 i}}{\lambda_{2 i}} e^{\lambda_{2 i}\left(\xi_{k}-1\right)}\right]+\frac{d_{3 i}}{\lambda_{3 i}}\left[2 e^{\lambda_{3 i} \xi_{k}}-1\right] \\
\leq & v_{i}\left(\xi_{k}\right)-\frac{\varepsilon^{\frac{5}{4}}}{4 r_{i}}\left(M_{1 i}+\left|v_{i}^{\prime \prime}(0)\right|+1\right) e^{-2 \sqrt{\frac{r_{i}}{\varepsilon}} \xi_{k}}+\frac{\varepsilon^{\frac{1}{2}}}{4 r_{i}}\left(M_{2 i}+\left|v_{i}^{\prime \prime}(1)\right|+1\right) e^{\frac{1}{2} \sqrt{\frac{r_{i}}{\varepsilon}}\left(\xi_{k}-1\right)} \\
& +\frac{c_{i}+M_{i}^{*}+1}{l_{i}}\left(2 e^{\frac{l_{i}+r_{i}}{r_{i}} \xi_{i}}-1\right) \varepsilon^{\frac{1}{5}} \\
\leq & v_{i}\left(\xi_{k}\right)+1 \leq\left|v_{i}\left(\xi_{k}\right)\right|+1:=\widetilde{m}_{i k}, \quad k=1,2, \ldots, m-2 .
\end{aligned}
$$

Similarly there exists $\widehat{\varepsilon}_{i j}>0$, for $0<\varepsilon \leq \widehat{\varepsilon}_{i j}(j=1,2, \ldots, n-2)$, we have

$$
\beta_{i}\left(\eta_{j}, \varepsilon\right) \leq\left|v_{i}\left(\eta_{j}\right)\right|+1:=\widehat{m}_{i j}, \quad j=1,2, \ldots, n-2 .
$$


Let

$$
\begin{aligned}
& M_{3 i}=\max _{k=1,2, \ldots, m-2}\left\{\widetilde{m}_{i k}\right\}, \quad M_{4 i}=\max _{j=1,2, \ldots, n-2}\left\{\widehat{m}_{i j}\right\}, \\
& \varepsilon_{0}=\min _{i=1,2, \ldots, N}\left\{\varepsilon_{i 1}, \varepsilon_{i 2}, \min _{k=1,2, \ldots, m-2}\left\{\widetilde{\varepsilon}_{i k}\right\}, \min _{j=1,2, \ldots, n-2}\left\{\widehat{\varepsilon}_{i j}\right\}\right\} .
\end{aligned}
$$

For $0<\varepsilon \leq \varepsilon_{0}$, we have $\boldsymbol{\beta}^{\prime}(0, \varepsilon) \succeq \mathbf{v}^{\prime}(0), \boldsymbol{\beta}^{\prime}(1, \varepsilon) \succeq \mathbf{v}^{\prime}(1), \boldsymbol{\beta}^{\prime \prime}(0, \varepsilon) \prec-\mathbf{M}_{1}, \boldsymbol{\beta}^{\prime \prime}(1, \varepsilon) \succeq \mathbf{M}_{2}$, $\boldsymbol{\beta}\left(\xi_{k}, \varepsilon\right) \preceq \mathbf{M}_{3}, \boldsymbol{\beta}\left(\eta_{j}, \varepsilon\right) \preceq \mathbf{M}_{4}, k=1,2, \ldots, m-2, j=1,2, \ldots, n-2$. Here $\mathbf{M}_{s}=\left(M_{s 1}, M_{s 2}, \ldots\right.$, $\left.M_{s N}\right)^{T}, s=1,2, \ldots, 6$. From condition (iii), we have

$$
\begin{aligned}
g_{i}\left(\boldsymbol{\beta}^{\prime}(0, \varepsilon), \boldsymbol{\beta}^{\prime \prime}(0, \varepsilon), \boldsymbol{\beta}\left(\xi_{1}, \varepsilon\right), \ldots, \boldsymbol{\beta}\left(\xi_{m-2}, \varepsilon\right)\right) & >g_{i}\left(\mathbf{v}^{\prime}(0),-\mathbf{M}_{1}, \mathbf{M}_{3}, \ldots, \mathbf{M}_{3}\right) \\
& \geq A_{i}, \\
h_{i}\left(\boldsymbol{\beta}^{\prime}(1, \varepsilon), \boldsymbol{\beta}^{\prime \prime}(1, \varepsilon), \boldsymbol{\beta}\left(\eta_{1}, \varepsilon\right), \ldots, \boldsymbol{\beta}\left(\eta_{m-2}, \varepsilon\right)\right) & \geq h_{i}\left(\mathbf{v}^{\prime}(1), \mathbf{M}_{2}, \mathbf{M}_{4}, \ldots, \mathbf{M}_{4}\right) \\
& \geq B_{i} .
\end{aligned}
$$

Thus $\boldsymbol{\beta}(t, \varepsilon)=\left(\beta_{1}(t, \varepsilon), \ldots, \beta_{N}(t, \varepsilon)\right)^{T}$ is an upper solution of SPBVP (1.1), (1.2). Similarly, we could show $\boldsymbol{\alpha}(t, \varepsilon)=\left(\alpha_{1}(t, \varepsilon), \ldots, \alpha_{N}(t, \varepsilon)\right)^{T}$ is a lower solution of SPBVP (1.1), (1.2). From Theorem 3.1, SPBVP (1.1), (1.2) has a solution $\mathbf{x}(t, \varepsilon)=\left(x_{1}(t, \varepsilon), \ldots, x_{N}(t, \varepsilon)\right)^{T}$ satisfying

$$
\boldsymbol{\alpha}(t, \varepsilon) \preceq \mathbf{x}(t, \varepsilon) \preceq \boldsymbol{\beta}(t, \varepsilon), \quad 0 \leq t \leq 1,
$$

and the inequality $(3.28)$ holds on $[0,1] \times\left[0, \varepsilon_{0}\right]$.

\section{Uniqueness result of SPBVP (1.1), (1.2)}

Theorem 4.1 Assume that all conditions of Theorem 3.2 hold, and for $i=1,2, \ldots, N$, the following inequalities hold:

$$
\begin{aligned}
& \bar{p}_{1 i}+\left(\sum_{k=1}^{m-2} \bar{\mu}_{k i}\right) \frac{r_{i}}{l_{i}}\left(2 e^{\frac{l_{i}+r_{i}}{r_{i}}}-1\right)>0, \\
& 2\left(\bar{p}_{2 i}+\frac{\bar{q}_{2 i} l_{i}}{r_{i}}\right) e^{\frac{r_{i}}{l_{i}}}+\left(\sum_{j=1}^{n-2} \bar{v}_{j i}\right) \frac{r_{i}}{l_{i}}\left(2 e^{\frac{l_{i}+r_{i}}{r_{i}}}-1\right)>0,
\end{aligned}
$$

where

$$
\begin{aligned}
& \bar{p}_{1 i}=\int_{0}^{1} g_{i z_{1 i}}\left(\mathbf{x}_{1}^{\prime}(0, \varepsilon)+\theta \mathbf{x}_{0}^{\prime}(0, \varepsilon), \mathbf{x}_{1}^{\prime \prime}(0, \varepsilon), \tau \mathbf{x}_{1}(t, \varepsilon)\right) d \theta, \\
& \bar{p}_{2 i}=\int_{0}^{1} h_{i z_{1 i}}\left(\mathbf{x}_{1}^{\prime}(1, \varepsilon)+\theta \mathbf{x}_{0}^{\prime}(1, \varepsilon), \mathbf{x}_{1}^{\prime \prime}(1, \varepsilon), \rho \mathbf{x}_{1}(t, \varepsilon)\right) d \theta, \\
& \bar{q}_{2 i}=\int_{0}^{1} h_{i z_{2 i}}\left(\mathbf{x}_{1}^{\prime}(1, \varepsilon), \mathbf{x}_{1}^{\prime \prime}(1, \varepsilon)+\theta \mathbf{x}_{0}^{\prime \prime}(1, \varepsilon), \rho \mathbf{x}_{1}(t, \varepsilon)\right) d \theta, \\
& \bar{\mu}_{k i}=\int_{0}^{1} g_{i z_{(k+2) i}}\left(\mathbf{x}_{1}^{\prime}(0, \varepsilon), \mathbf{x}_{1}^{\prime \prime}(0, \varepsilon), \tau \mathbf{x}_{1}(t, \varepsilon)+\theta \mathbf{x}_{0}\left(\xi_{k}, \varepsilon\right)\right) d \theta, \quad k=1,2, \ldots, m-2, \\
& \bar{v}_{j i}=\int_{0}^{1} h_{i z(j+2) i}\left(\mathbf{x}_{1}^{\prime}(1, \varepsilon), \mathbf{x}_{1}^{\prime \prime}(1, \varepsilon), \rho \mathbf{x}_{1}(t, \varepsilon)+\theta \mathbf{x}_{0}\left(\eta_{j}, \varepsilon\right)\right) d \theta, \quad j=1,2, \ldots, n-2,
\end{aligned}
$$




$$
\begin{aligned}
& \tau \mathbf{x}_{1}(t, \varepsilon):=\left(\mathbf{x}_{1}\left(\xi_{1}, \varepsilon\right), \mathbf{x}_{1}\left(\xi_{2}, \varepsilon\right), \ldots, \mathbf{x}_{1}\left(\xi_{m-2}, \varepsilon\right)\right), \\
& \rho \mathbf{x}_{1}(t, \varepsilon):=\left(\mathbf{x}_{1}\left(\eta_{1}, \varepsilon\right), \mathbf{x}_{1}\left(\eta_{2}, \varepsilon\right), \ldots, \mathbf{x}_{1}\left(\eta_{n-2}, \varepsilon\right)\right), \\
& \tau \mathbf{x}_{1}(t, \varepsilon)+\theta \mathbf{x}_{0}\left(\xi_{k}, \varepsilon\right):=\left(\mathbf{x}_{1}\left(\xi_{1}, \varepsilon\right), \ldots, \mathbf{x}_{1}\left(\xi_{k}, \varepsilon\right)+\theta \mathbf{x}_{0}\left(\xi_{k}, \varepsilon\right), \ldots, \mathbf{x}_{1}\left(\xi_{m-2}, \varepsilon\right)\right), \\
& \rho \mathbf{x}_{1}(t, \varepsilon)+\theta \mathbf{x}_{0}\left(\eta_{j}, \varepsilon\right):=\left(\mathbf{x}_{1}\left(\eta_{1}, \varepsilon\right), \ldots, \mathbf{x}_{1}\left(\eta_{j}, \varepsilon\right)+\theta \mathbf{x}_{0}\left(\eta_{j}, \varepsilon\right), \ldots, \mathbf{x}_{1}\left(\eta_{n-2}, \varepsilon\right)\right), \\
& g_{i z_{k i}}=\frac{\partial g_{i}\left(\mathbf{z}_{1}, \mathbf{z}_{2}, \ldots, \mathbf{z}_{m}\right)}{\partial z_{k i}}, \quad k=1,2, \ldots, m, \\
& h_{i z_{j i}}=\frac{\partial h_{i}\left(\mathbf{z}_{1}, \mathbf{z}_{2}, \ldots, \mathbf{z}_{n}\right)}{\partial z_{j i}}, \quad j=1,2, \ldots, n,
\end{aligned}
$$

and $l_{i}, r_{i}, i=1,2, \ldots, N$ are given in Theorem 3.2. Then SPBVP (1.1), (1.2) has a unique solution.

Proof From Theorem 3.2, for SPBVP (1.1), (1.2) there exist solutions. In order to show the uniqueness of the solutions, we only need to show (1.1), (1.2) has at most one solution. If the assertion is not true, then $\operatorname{SPBVP}(1.1),(1.2)$ has two different solutions $\mathbf{x}_{1}(t, \varepsilon), \mathbf{x}_{2}(t, \varepsilon)$. Let

$$
\mathbf{y}(t, \varepsilon)=\mathbf{x}_{2}(t, \varepsilon)-\mathbf{x}_{1}(t, \varepsilon)
$$

then $\mathbf{y}(t, \varepsilon)$ is a solution of the boundary value problem

$$
\begin{aligned}
& \varepsilon \mathbf{x}^{\prime \prime \prime}(t, \varepsilon)+\bar{\rho}_{1}(t, \varepsilon) \mathbf{x}^{\prime \prime}(t, \varepsilon)+\bar{\rho}_{2}(t, \varepsilon) \mathbf{x}^{\prime}(t, \varepsilon)+\bar{\rho}_{3}(t, \varepsilon) \mathbf{x}(t, \varepsilon)=\mathbf{0}, \quad 0 \leq t \leq 1, \\
& \left\{\begin{array}{l}
\mathbf{x}(0, \varepsilon)=0 \\
\bar{P}_{1} \mathbf{x}^{\prime}(0, \varepsilon)+\bar{Q}_{1} \mathbf{x}^{\prime \prime}(0, \varepsilon)+\sum_{k=1}^{m-2} \bar{\mu}_{k} \mathbf{x}\left(\xi_{k}, \varepsilon\right)=\mathbf{0}, \\
\bar{P}_{2} \mathbf{x}^{\prime}(1, \varepsilon)+\bar{Q}_{2} \mathbf{x}^{\prime \prime}(1, \varepsilon)+\sum_{j=1}^{n-2} \bar{v}_{j} \mathbf{x}\left(\eta_{i}, \varepsilon\right)=\mathbf{0},
\end{array}\right.
\end{aligned}
$$

where $\bar{\rho}_{s}(t, \varepsilon)=\operatorname{diag}\left(\bar{\rho}_{s 1}(t, \varepsilon), \ldots, \bar{\rho}_{s N}(t, \varepsilon)\right), s=1,2,3, \bar{P}_{1}=\operatorname{diag}\left(\bar{p}_{11}, \bar{p}_{12}, \ldots, \bar{p}_{1 N}\right), \bar{P}_{2}=$ $\operatorname{diag}\left(\bar{p}_{21}, \bar{p}_{22}, \ldots, \bar{p}_{2 N}\right), \quad \bar{Q}_{1}=\operatorname{diag}\left(\bar{q}_{11}, \bar{q}_{12}, \ldots, \bar{q}_{1 N}\right), \quad \bar{Q}_{2}=\operatorname{diag}\left(\bar{q}_{21}, \bar{q}_{22}, \ldots, \bar{q}_{2 N}\right), \quad \bar{\mu}_{k}=$ $\operatorname{diag}\left(\bar{\mu}_{k 1}, \ldots, \bar{\mu}_{k N}\right), \bar{v}_{j}=\operatorname{diag}\left(\bar{v}_{j 1}, \ldots, \bar{v}_{j N}\right), k=1,2, \ldots, m-2, j=1,2, \ldots, n-2$,

$$
\begin{aligned}
& \bar{\rho}_{1 i}(t, \varepsilon)=\int_{0}^{1} f_{i x_{i}^{\prime \prime}}\left(t, \mathbf{x}_{1}(t, \varepsilon), \mathbf{x}_{1}^{\prime}(t, \varepsilon), \mathbf{x}_{1}^{\prime \prime}(t, \varepsilon)+\theta \mathbf{y}^{\prime \prime}(t, \varepsilon), \varepsilon\right) d \theta, \\
& \bar{\rho}_{2 i}(t, \varepsilon)=\int_{0}^{1} f_{i x_{i}^{\prime}}\left(t, \mathbf{x}_{1}(t, \varepsilon), \mathbf{x}_{1}^{\prime}(t, \varepsilon)+\theta \mathbf{y}^{\prime}(t, \varepsilon), \mathbf{x}_{1}^{\prime \prime}(t, \varepsilon), \varepsilon\right) d \theta, \\
& \bar{\rho}_{3 i}(t, \varepsilon)=\int_{0}^{1} f_{i x_{i}}\left(t, \mathbf{x}_{1}(t, \varepsilon)+\theta \mathbf{y}(t, \varepsilon), \mathbf{x}_{1}^{\prime}(t, \varepsilon), \mathbf{x}_{1}^{\prime \prime}(t, \varepsilon), \varepsilon\right) d \theta, \\
& \bar{q}_{1 i}=\int_{0}^{1} g_{i z_{2 i}}\left(\mathbf{x}_{1}^{\prime}(0, \varepsilon), \mathbf{x}_{1}^{\prime \prime}(0, \varepsilon)+\theta \mathbf{x}_{0}^{\prime \prime}(0, \varepsilon), \tau \mathbf{x}_{1}(t, \varepsilon)\right) d \theta .
\end{aligned}
$$

From conditions (ii), (iii) in Theorem 3.2, we obtain $\bar{\rho}_{s i} \in C\left([0,1] \times\left[0, \varepsilon_{0}\right], R\right), s=1,2,3$ and $\bar{\rho}_{1 i}(t, \varepsilon) \leq 0, \bar{\rho}_{2 i}(t, \varepsilon) \leq-r_{i}<0,0 \leq \bar{\rho}_{3 i}(t, \varepsilon) \leq l_{i},(t, \varepsilon) \in[0,1] \times\left[0, \varepsilon_{0}\right]$, and $\bar{q}_{1 i} \leq 0, \bar{q}_{2 i} \geq 0$, $\bar{\mu}_{k i} \leq 0, \bar{v}_{j i} \leq 0, i=1,2, \ldots, N, k=1,2, \ldots, m-2, j=1,2, \ldots, n-2$. That is, $\bar{\rho}_{s}(t, \varepsilon), s=1,2,3$, $\bar{Q}_{1}, \bar{Q}_{2}, \bar{\mu}_{k}, \bar{v}_{j}$, satisfy Eq. (2.4) and boundary conditions (2.5). 
Define

$$
\phi_{i}(t, \varepsilon)=\frac{2 e^{\lambda_{3 i} t}-1}{\lambda_{3 i}}-\frac{2 \lambda_{3 i} e^{\lambda_{1 i} t}}{\lambda_{1 i}^{2}} .
$$

It is obvious that $\phi_{i}(t, \varepsilon)>0, \phi_{i}^{\prime}(t, \varepsilon)>0, \phi_{i}^{\prime \prime}(t, \varepsilon) \geq 0$, and

$$
\begin{aligned}
& \varepsilon \phi_{i}^{\prime \prime \prime}(t, \varepsilon)+\bar{\rho}_{1 i}(t, \varepsilon) \phi_{i}^{\prime \prime}(t, \varepsilon)+\bar{\rho}_{2 i}(t, \varepsilon) \phi_{i}^{\prime}(t, \varepsilon)+\bar{\rho}_{3 i}(t, \varepsilon) \phi_{i}(t, \varepsilon) \\
& \quad \leq \varepsilon \phi_{i}^{\prime \prime \prime}(t, \varepsilon)-r_{i} \phi_{i}^{\prime}(t, \varepsilon)+l_{i} \phi_{i}(t, \varepsilon) \\
& \quad=\frac{2}{\lambda_{3 i}} e^{\lambda_{3 i} t}\left(\varepsilon \lambda_{3 i}^{3}-r_{i} \lambda_{3 i}+l_{i}\right)-\frac{2 \lambda_{3 i}}{\lambda_{1 i}^{2}} e^{\lambda_{1 i} t}\left(\varepsilon \lambda_{1 i}^{3}-r_{i} \lambda_{1 i}+l_{i}\right)-\frac{l_{i}}{\lambda_{3 i}} \\
& =-\frac{l_{i}}{\lambda_{3 i}}<0 .
\end{aligned}
$$

For $0<\varepsilon \leq \varepsilon_{0}$, from (4.1), (4.2), we have

$$
\begin{aligned}
& \bar{p}_{1 i} \phi_{i}^{\prime}(0, \varepsilon)+\bar{q}_{1 i} \phi_{i}^{\prime \prime}(0, \varepsilon)+\sum_{k=1}^{m-2} \bar{\mu}_{k i} \phi_{i}\left(\xi_{k i}, \varepsilon\right) \\
& =2 \bar{p}_{1 i}\left(1-\frac{\lambda_{3 i}}{\lambda_{1 i}}\right)+\sum_{k=1}^{m-2} \bar{\mu}_{k i}\left(\frac{2 e^{\lambda_{3 i} \xi_{k i}}-1}{\lambda_{3 i}}-\frac{2 \lambda_{3 i} e^{\lambda_{1 i} \xi_{k i}}}{\lambda_{1 i}^{2}}\right) \\
& \geq \bar{p}_{1 i}+\sum_{k=1}^{m-2} \bar{\mu}_{k i} \frac{r_{i}}{l_{i}}\left(2 e^{\frac{l_{i}+r_{i}}{r_{i}} \xi_{k i}}-1\right) \\
& \geq \bar{p}_{1 i}+\left(\sum_{k=1}^{m-2} \bar{\mu}_{k i}\right) \frac{r_{i}}{l_{i}}\left(2 e^{\frac{l_{i}+r_{i}}{r_{i}}}-1\right)>0, \\
& \bar{p}_{2 i} \phi_{i}^{\prime}(1, \varepsilon)+\bar{q}_{2 i} \phi_{i}^{\prime \prime}(1, \varepsilon)+\sum_{j=1}^{n-2} \bar{v}_{j i} \phi_{i}\left(\eta_{j}, \varepsilon\right) \\
& \geq 2\left(\bar{p}_{2 i}+\frac{\bar{q}_{2 i} l_{i}}{r_{i}}\right) e^{\frac{r_{i}}{l_{i}}}+\sum_{j=1}^{n-2} \bar{v}_{j i} \frac{r_{i}}{l_{i}}\left(2 e^{\frac{l_{i}+r_{i}}{r_{i}} \eta_{j i}}-1\right) \\
& \geq 2\left(\bar{p}_{2 i}+\frac{\bar{q}_{2 i} l_{i}}{r_{i}}\right) e^{\frac{r_{i}}{l_{i}}}+\left(\sum_{j=1}^{n-2} \bar{v}_{j i}\right) \frac{r_{i}}{l_{i}}\left(2 e^{\frac{l_{i}+r_{i}}{r_{i}}}-1\right)>0
\end{aligned}
$$

Then $\boldsymbol{\Phi}(t, \varepsilon)=\left(\phi_{1}(t, \varepsilon), \ldots, \phi_{N}(t, \varepsilon)\right)^{T}$ satisfies the conditions in Lemma 2.1. Hence SPBVP (4.3), (4.4) has only a zero solution, which contradicts $\mathbf{x}_{1}(t, \varepsilon) \neq \mathbf{x}_{2}(t, \varepsilon)$. Therefore, SPBVP (1.1), (1.2) has a unique solution.

Remark 4.1 If we take $N=1$, we find that SPBVP (1.1), (1.2) becomes the singularly perturbed boundary value problem (3), (4) in [10]. It is notable that our results agree well with the corresponding ones in [10]. 
Remark 4.2 If we choose $N=1, m=n$, and take the nonlinear boundary functions $g, h$ to occur in the following linear functions:

$$
\begin{aligned}
& g\left(x_{1}, x_{2}, \ldots, x_{n}\right)=a x_{1}-b x_{2}+\sum_{i=3}^{n} \alpha_{i} x_{i}, \\
& h\left(y_{1}, y_{2}, \ldots, y_{n}\right)=c y_{1}+d y_{2}+\sum_{j=3}^{n} \beta_{j} y_{j},
\end{aligned}
$$

then SPBVP (1.1), (1.2) becomes the singularly perturbed boundary value problem (1.1), (1.2) in [9].

Remark 4.3 If we choose the nonlinear boundary functions $\mathbf{G}, \mathbf{H}$ to be the following linear functions:

$$
\mathbf{G}=P_{1} \mathbf{x}^{\prime}(0, \varepsilon)-P_{2} \mathbf{x}^{\prime \prime}(0, \varepsilon), \quad \mathbf{H}=Q_{1} \mathbf{x}^{\prime}(1, \varepsilon)-Q_{2} \mathbf{x}^{\prime \prime}(1, \varepsilon)
$$

then SPBVP (1.1), (1.2) becomes the singularly perturbed boundary value problem (1), (2) in [20]. In this paper, we get the existence and uniqueness of solutions. We also discuss the asymptotic estimates of solutions.

\section{Acknowledgements}

The authors express their sincere thanks to the anonymous referees for corrections of the paper and suggestions for improving the quality of the paper.

\section{Funding}

This work is supported by the Natural Science Foundation of China (Grant Nos. 11771185, 11871251 and 11801231).

Availability of data and materials

Data sharing not applicable to this article as no datasets were generated or analysed during the current study.

\section{Competing interests}

The authors declare that they have no competing interests.

\section{Authors' contributions}

All authors contributed equally to the writing of this paper. All authors read and approved the final manuscript.

\section{Publisher's Note}

Springer Nature remains neutral with regard to jurisdictional claims in published maps and institutional affiliations.

Received: 29 July 2019 Accepted: 8 January 2020 Published online: 21 January 2020

\section{References}

1. Zhang, Y., Bai, Z., Feng, T.: Existence results for a coupled system of nonlinear fractional three-point boundary value problems at resonance. Comput. Math. Appl. 61, 1032-1047 (2011)

2. Du, Z., Zhao, B., Bai, Z.: Solvability of a third order multipoint boundary value problem at resonance. Abstr. Appl. Anal. 2014, Article ID 931217 (2014)

3. Bai, Z., Chen, Y., Lian, H., et al.: On the existence of blow up solutions for a class of fractional differential equations. Fract. Calc. Appl. Anal. 17, 1175-1187 (2014)

4. Lin, X., Liu, J., Wang, C.: The existence and asymptotic estimates of solutions for a third-order nonlinear singularly perturbed boundary value problem. Qual. Theory Dyn. Syst. 18, 687-710 (2019)

5. Zhao, W.: Singular perturbations of boundary value problems for a class of third-order nonlinear ordinary differential equations. J. Differ. Equ. 88, 265-278 (1990)

6. Valarmathi, S., Ramanujam, N.: An asymptotic numerical method for singular perturbed third-order differential equations of convection-diffusion type. Comput. Math. Appl. 44, 693-710 (2002)

7. Alinia, N., Zarebnia, M.: A new tension B-spline method for third-order self-adjoint singularly perturbed boundary value problems. J. Comput. Appl. Math. 342, 521-533 (2018)

8. Howes, F.A.: The asymptotic solution of a class of third-order boundary value problem arising in the theory of thin film flow. SIAM J. Appl. Math. 43, 993-1004 (1983) 
9. Du, Z., Ge, W., Zhou, M.: Singular perturbations for third-order nonlinear multi-point boundary value problem. J. Differ. Equ. 218(1), 69-90 (2005)

10. Lin, X: Singular perturbations of third-order nonlinear differential equations with full nonlinear boundary conditions. Appl. Math. Comput. 224, 88-95 (2013)

11. O'Malley, R.E.: Singular Perturbation Methods for Ordinary Differential Equations. Springer, New York (1991)

12. Lodhi, R.K., Mishra, H.K.: Quintic B-spline method for solving second order linear and nonlinear singularly perturbed two-point boundary value problems. J. Comput. Appl. Math. 319, 170-187 (2017)

13. $\mathrm{Xu}, \mathrm{Y} ., \mathrm{Du}, \mathrm{Z} ., \mathrm{W}$ ei, L.: Geometric singular perturbation method to the existence and asymptotic behavior of traveling waves for a generalized Burgers-KdV equation. Nonlinear Dyn. 83, 65-73 (2016)

14. Du, Z., Feng, Z.: Existence and asymptotic behavior of traveling waves in a modified vector-disease model. Commun. Pure Appl. Anal. 17, 1899-1920 (2018)

15. Chen, A., Guo, L.N., Deng, X.: Existence of solitary waves and periodic waves for a perturbed generalized BBM equations. J. Differ. Equ. 261, 5324-5349 (2016)

16. Du, Z., Li, J., Li, X.: The existence of solitary wave solutions of delayed Camassa-Holm via a geometric approach. J. Funct. Anal. 275, 988-1007 (2018)

17. Ge, J., Du, Z: The solitary wave solutions of the nonlinear perturbed shallow water wave model. Appl. Math. Lett. 103 106202 (2020). https://doi.org/10.1016/j.aml.2019.106202

18. Campana, C., Meziani, A.: Boundary value problems for a class of planar complex vector fields. J. Differ. Equ. 261, 5609-5636 (2016)

19. Andres, J., Malaguti, L., Pavlačková, M.: Strictly localized bounding functions for vector second-order boundary value problems. Nonlinear Anal. 71, 6019-6028 (2009)

20. Du, Z.: Singularly perturbed third-order boundary value problem for nonlinear systems. Appl. Math. Comput. 189 869-877 (2007)

21. Wang, G., Zhou, M., Sun, L.: Bounding functions methods for fully nonlinear boundary value problems. Nonlinear Anal. 64, 696-705 (2006)

\section{Submit your manuscript to a SpringerOpen ${ }^{\circ}$ journal and benefit from:}

- Convenient online submission

- Rigorous peer review

- Open access: articles freely available online

- High visibility within the field

- Retaining the copyright to your article

Submit your next manuscript at $\boldsymbol{~ s p r i n g e r o p e n . c o m ~}$ 